\title{
Geometric and Analytic Views in Existence Theorems for Optimal Control. III. Weak Solutions ${ }^{1}$
}

\author{
L. CeSARI ${ }^{2}$
}

\begin{abstract}
Existence theorems are proved for weak optimal solutions of problems of optimization with distributed and boundary control. Many examples are given. Application is made of recent remarks on closure properties of linear and nonlinear operators. Recent geometric, topological, and analytical views are brought to bear on the underlying seminormality conditions.
\end{abstract}

Key Words. Existence theorems, weak solutions, distributed and boundary controls, seminormality, analytic criteria, closed operators.

\section{Introduction}

In the present paper, we discuss optimal solutions of problems of optimization with distributed and boundary controls, with application of recent remarks on closure properties of linear and nonlinear operators, and geometric, topological, and analytic views concerning seminormality conditions. The latter type of requirements are indeed drastically reduced or completely eliminated in the present paper.

The present paper completes the previous ones (Refs. 1,2) where we limited ourselves to strong solutions only. For the sake of brevity, we refer to Ref. 2 for some of the notations and definitions.

\section{Problem of Optimization with State Equations in the Weak Form}

As pointed out in Ref. 2, we are interested in the minimum of functionals of the form

$$
I=\sum_{j=1}^{N} \int_{G_{j}} F_{j} d \mu_{j}
$$

\footnotetext{
${ }^{1}$ This research was partially supported by AFOSR Research Project 71-2122 at the University of Michigan, Ann Arbor, Michigan.

${ }^{2}$ Professor, Department of Mathematics, University of Michigan, Ann Arbor, Michigan.
} 
on measure spaces $\left(G_{j}, A_{j}, \mu_{j}\right), j=1, \ldots, N$, under constraints of the form $\mathscr{L}_{j} x=N_{j}\left[x, u_{j}\right]$, each involving a certain $\mu_{j}$-measurable control function $u_{j}$ on $G_{j}$, while the unknown $x$ is an element of some abstract topological space $X$. As in Ref. 2, to be specific and for the sake of simplicity, we assume that one of the spaces is a subset $G$ of $E^{\nu}$ of point $t$, with Lebesgue measure $d t$, and a functional relation $\mathscr{L} x=N[x, u]$ to satisfy, involving a measurable control function $u$ with values in $E^{m}$, and that there is only another space, namely, a measure space $(\Gamma, \mathscr{A}, \mu)$ of points $\tau$, with measure $\mu$, and a functional relation to satisfy $\mathscr{I} x=N^{\prime}[x, v]$, involving a $\mu$-measurable control function $v$ with values in $E^{m^{\prime}}$. Actually, in most applications and examples, $G$ is a Morrey-type domain, $\Gamma$ is a part of the boundary of $G$ of some dimension $1 \leq \sigma \leq \gamma-1$, and $\mu$ is simply the $\sigma$-measure (area, length, or in general $\sigma$-area subsumed by $G$ on $\Gamma$ ). Then, $X$ is a Sobolev space $W_{p}^{l}(G)$ on $G$, and Sobolev's imbedding theorems may have a fundamental role.

In the present paper, we are interested in the case where the functional relations on $G$ and on $\Gamma$ may be written in weak form as is usual in partial differential equation theory.

Let $G$ be any measurable subset of $E^{\nu}$ with finite Lebesgue measure $|G|<\infty$. For every point $t \in G$, let $A(t)$ denote a given subset of $E^{s}$ and let $A$ denote the set

$$
A=[(t, y) \mid t \in G, y \in A(t)] .
$$

For every $(t, y) \in A$, let $U(t, y)$ denote a given subset of $E^{m}$, and let $M$ denote the set

$$
M=[(t, y, u) \mid t \in G, y \in A(t), u \in U(t, y)] \subset E^{\nu+s+m} .
$$

Let $f_{0}(t, y, u), f(t, y, u)=\left(f_{1}, \ldots, f_{r}\right)$ denote given functions defined on $M$ with values on $E^{1}$ and $E^{r}$, respectively.

Let $(\Gamma, d)$ be a given metric space which is also a finite complete measure space $(\Gamma, \mathscr{A}, \mu)$ such that the $\sigma$-algebra $\mathscr{A}$ contains the Borel sets of $(\Gamma, d)$. For every point $\tau \in \Gamma$, let $B(\tau)$ denote a given subset of $E^{s^{\prime}}$, and let $B$ denote the set

$$
B=[(\tau, \dot{y}) \mid \tau \in \Gamma, \dot{y} \in B(\tau)] .
$$

For every $(\tau, \dot{y}) \in B$, let $V(\tau, \dot{y})$ denote a given subset of $E^{m^{\prime}}$, and let $\stackrel{\circ}{M}$ denote the set

$$
\stackrel{\circ}{M}=[(\tau, \dot{y}, v) \mid \tau \in \Gamma, \stackrel{o}{y} \in B(\tau), v \in V(\tau, \dot{y})] \subset \Gamma \times E^{s^{\prime}+m^{\prime}} .
$$

Let $g_{0}(\tau, \stackrel{\circ}{y}, v), g(\tau, \stackrel{\circ}{y}, v)=\left(g_{1}, \ldots, g_{r^{\prime}}\right)$ denote given functions defined on $\dot{M}$ with values in $E^{1}$ and $E^{r^{\prime}}$, respectively.

Here, $y, \stackrel{o}{y}$ are the state variables, $u$ and $v$ the control variables, the sets $U(t, y), V(\tau, \stackrel{y}{y})$ the control spaces, on $G$ and on $\Gamma$, respectively. 
We shall assume that $f_{0}, f, A, M$ satisfy a Carathéodory condition (C), as stated in Ref. 2. Thus, in particular, $f_{0}, f$ are measurable in $t$ for every $(y, u)$ and continuous in $(y, u)$ for almost all $t$. Analogously, we assume that $g_{0}, g$, $B, \stackrel{M}{M}$ satisfy a Carathéodory condition (C).

Let $(X, \mathscr{T})$ be a given topological space and $S$ a given subset of $X$. Let $\mathcal{M}$ and $\mathscr{K}$ be operators, not necessarily linear, mapping $S$ into $\left(L_{p}(G)\right)^{r}$ and $\left(L_{p}(\Gamma)\right)^{r^{\prime}}$, respectively, $1 \leq p \leq \infty$.

To state the general weak form of the state equations, we need first the space $W$ of test functions $w=\left(w_{1}, w_{2}\right)$,

$$
W \subset\left(L_{q}(G)\right)^{r} \times\left(L_{q}(\Gamma)\right)^{r^{\prime}},
$$

with

$$
p^{-1}+q^{-1}=1, \quad 1 \leq q \leq \infty, \quad 1 \leq p \leq \infty .
$$

Namely, we shall assume that $W$ is a normed space with norm \|\|$_{W}$ satisfying a relation

$$
\left\|w_{1}\right\|_{q}+\left\|w_{2}\right\|_{q} \leq K\|w\|_{w} \quad \text { for all } w=\left(w_{1}, w_{2}\right) \in W,
$$

and some constant $K$, where \|\|$_{q}$ are the $L_{q}$-norm in $G$ or $\Gamma$. Let $W^{*}$ denote the dual space of $W$, so that

$$
W^{*} \supset\left(L_{p}(G)\right)^{r} \times\left(L_{p}(\Gamma)\right)^{r^{\prime}} .
$$

Let $T$ denote the set of all measurable functions $u: G \rightarrow E^{m}$ and $\stackrel{T}{T}$ the set of all $\mu$-measurable functions $v: \Gamma \rightarrow E^{m^{\prime}}$. Let $\mathscr{F}: S \rightarrow W^{*}$ be a given operator, not necessarily linear; and, for any $(x, u, v), x \in S, u \in T, v \in \stackrel{\circ}{T}$ [under the usual restrictions $\mathscr{M} x(t) \in A(t), u(t) \in U(t, \mathcal{M} x(t)), t \in G, \mathscr{K} x(\tau) \epsilon$ $B(\tau), v(\tau) \in V(\tau, \mathscr{K} x(\tau)), \tau \in \Gamma]$, let

$$
f(t, \mathcal{M} x(t), u(t)) \in L_{p}(G), \quad g(\tau, \mathscr{K} x(\tau), v(\tau)) \in\left(L_{p}(\Gamma)\right),
$$

let $h$, or $h_{x u v}$, or $h(x, u, v)$ be the linear operator $h: W \rightarrow E^{1}$, defined by

$$
\begin{aligned}
h_{x u v} w=h\left(w_{1}, w_{2}\right)=\int_{G} f(t, \mathcal{M} x(t), u(t)) \cdot & w_{1}(t) d t \\
& \quad+\int_{\Gamma} g(\tau, \mathscr{H} x(\tau), v(\tau)) \cdot w_{2}(\tau) d \mu,
\end{aligned}
$$

where $f \cdot w_{1}$ and $g \cdot w_{2}$ denote usual inner products in $E^{r}$ and $E^{r^{\prime}}$, respectively. The weak form of the state equation is now

$$
\mathscr{F}=h,
$$

or

$$
\mathscr{F}(x) w=h(x, u, v) w,
$$


or

$$
\mathscr{F}(x)\left(w_{1}, w_{2}\right) h_{x u v}\left(w_{1}, w_{2}\right) \quad \text { for all } w \in W
$$

We are now in a position to state the problem of optimization we are concerned with in the present paper. Namely, we are concerned with the

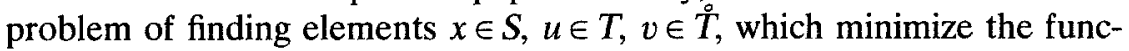
tional

$$
I[x, u, v]=\int_{G} f_{0}(t, \mathcal{M} x(t), u(t)) d t+\int_{\Gamma} g_{0}(\tau, \mathscr{H} x(\tau), v(\tau)) d \mu
$$

with state equation

$$
\mathscr{F} w=h(x, u, v) w \quad \text { for all } w \in W,
$$

and constraints

$$
\begin{array}{cccc}
\mathscr{M} x(t) \in A(t), & u(t) \in U(t, \mathscr{M} x(t)), & t \in G, & \text { a.e., } \\
\mathscr{K} x(\tau) \in B(\tau), & v(\tau) \in V(\tau, \mathscr{K} x(\tau)), & \tau \in \Gamma, & \mu \text {-a.e. }
\end{array}
$$

The only functional relation, Eq. (3), replaces here the two strong form state equations usually written as

$$
(\mathscr{L} x)(t)=f(t,(\mathcal{M} x)(t), u(t)) \quad \text { in } G
$$

and

$$
(\mathscr{I} x)(t)=g(\tau,(\mathscr{K} x)(\tau), v(\tau)) \quad \text { on } \Gamma
$$

as in Ref. 2. In any particular situation, it is understood that Eq. (3) is a generalization of the corresponding strong form, [that is, whenever $x, u, v$ satisfy $\mathscr{L} x=f, \mathscr{F} x=g$, then they satisfy necessarily Eq. (3)]. Many examples have already been given in Ref. 3. In Ref. 3, we showed also that the case where controls appear in $\mathscr{L}$ and $\mathscr{F}$, and therefore in $\mathscr{F}$, does not present difficulties (see Remark 3.1 below and examples).

For every $t \in G$ and $y \in A(t)$, we shall denote by $\tilde{Q}(t, y)$ the set

$$
\tilde{Q}(t, y)=\left[\left(z^{0}, z\right) \mid z^{0} \geq f_{0}(t, y, u), z=f(t, y, u), u \in U(t, y)\right] \subset E^{r+1} .
$$

For every $\tau \in \Gamma$ and $\dot{y} \in B(\tau)$, we shall denote by $\tilde{R}(\tau, \dot{y})$ the set

$$
\tilde{R}(\tau, \dot{y})=\left[\left(z^{o}, z\right) \mid z^{0} \geq g_{0}(\tau, \dot{y}, v), z=g(\tau, \dot{y}, v), v \in V(\tau, \dot{y})\right] \subset E^{r^{\prime}+1} .
$$

As in Ref. 2, we need properties of the sets $\tilde{Q}(t, y), \tilde{\tilde{R}}(t, \stackrel{\circ}{y})$. We restate here only properties $(\mathrm{K}),(\mathrm{Q}),(\mathrm{P})$, and $\left(\mathrm{P}^{\prime}\right)$ with respect to $y$ for the sets $\tilde{Q}(t, y)$. 
Topological Properties (K) and (Q). The sets $\tilde{Q}(t, y)$ are said to satisfy property $(\mathrm{K})$ at $\left(t_{0}, y_{0}\right)$ with respect to $y$ provided

$$
\tilde{Q}\left(t_{0}, y_{0}\right)=\bigcap_{\epsilon>0} \mathrm{cl} \bigcup_{u \in N_{\varepsilon}\left(y_{0}\right)} \tilde{Q}\left(t_{0}, y\right),
$$

where

$$
N_{\epsilon}\left(y_{0}\right)=\left[y \in A(t),\left|y-y_{0}\right| \leq \epsilon\right] .
$$

The same sets are said to satisfy property $(\mathrm{Q})$ at $\left(t_{0}, y_{0}\right)$ with respect to $y$ (Ref. 4) provided

$$
\tilde{Q}\left(t_{0}, y_{0}\right)=\bigcap_{\epsilon>0} \operatorname{clco} \bigcup_{y \in N_{\varepsilon}\left(y_{0}\right)} \tilde{Q}\left(t_{0}, y\right) \text {. }
$$

Any set $Q(t, y)$ satisfying property $(\mathrm{K})$ is closed; any set satisfying property $(\mathrm{Q})$ is closed and convex.

A number of criteria have been proved in Refs. 5-8, which guarantee property $(\mathrm{Q})$ of the sets $\tilde{Q}(t, y)$. We mention here only one which we shall need below.

For any given $t_{0} \in G$ and $y_{0} \in A\left(t_{0}\right)$, a function $\psi\left(t_{0}, y, u\right)$ is said to be of slow growth with respect to $f_{0}(t, y, u)$ at $y_{0}$, provided

(a) for some $\delta>0$ and $M \geq 0$, we have

$$
f_{0}\left(t_{0}, y, u\right) \geq-M \quad \text { for all } y \in A\left(t_{0}\right), \quad\left|y-y_{0}\right| \leq \delta, \quad u \in U\left(t_{0}, y\right),
$$
that

(b) given $\epsilon>0$, there is $\bar{u} \geq 0$ (which may depend on $\epsilon, \delta, M, t_{0}, y_{0}$ ) such

$$
y \in A\left(t_{0}\right), \quad\left|y-y_{0}\right| \leq \delta, \quad u \in U\left(t_{0}, y\right), \quad|u| \geq \bar{u}
$$

implies

$$
\left|\psi\left(t_{0}, y, u\right)\right| \leq \epsilon\left[f_{0}\left(t_{0}, y, u\right)+M\right]
$$

It was proved in Ref. 4 under different notations that, if $f_{0}\left(t_{0}, y, u\right)$, $f\left(t_{0}, y, u\right)$ are continuous in $(y, u)$, and both 1 and $f\left(t_{0}, y, u\right)$ are of slow growth with respect to $f_{0}\left(t_{0}, y, u\right)$ at $y_{0}$, then the sets $\tilde{Q}\left(t_{0}, y\right)$ if convex, have property (Q) with respect to $y$ at $\left(t_{0}, y_{0}\right)$.

Geometrical Property (P). First, let us note that the projection of the set $\tilde{Q}(t, y)$ on the $z$-space $E^{r}$ is the set

$$
Q(t, y)=[z \mid z=f(t, y, u), u \in U(t, y)] \subset E^{r},
$$

and thus $Q(t, y)$ is convex whenever $\tilde{Q}(t, y)$ is convex. For every $t \in G$, $y \in A(t), z \in Q(t, y)$, we denote by $T(z ; t, y)$ the scalar function

$$
T(z ; t, y)=\inf \left[z^{0} \mid\left(z^{0}, z\right) \in \tilde{Q}(t, y)\right], \quad-\infty \leq T(z ; t, y)<+\infty .
$$

For every $N>0$, we denote by $V(0, N)$ the closed ball $\left[z \in E^{r}|| z \mid \leq N\right]$. 
We say that the sets $\tilde{Q}(t, y)$ satisfy property (P) with respect to $y$ provided

$\left(\mathrm{P}_{1}\right)$ there is a measurable bounded function $\tilde{p}(t)=\left(p^{0}(t), p(t)\right), t \in G$, $p^{0}(t) \in E^{1}, p(t) \in E^{r}$, say $\left|p^{0}(t)\right| \leq c,|p(t)| \leq \sigma, t \in G$, such that $\tilde{p}(t) \in \tilde{Q}(t, y)$, $T(z ; t, y) \geq-c$ for all $(t, y) \in A, t \in G-T_{0},\left|T_{0}\right|=0$, and $z \in Q(t, y)$ with $|z| \geq \sigma$,

$\left(\mathrm{P}_{2}\right)$ for every $N>\sigma$, the sets $\tilde{Q}(t, y) \cap\left(E^{1} \times V(0, N)\right)$ have property (Q) with respect to $y$ at every $\left(t_{0}, y\right) \in A, t_{0} \in G-T_{0},\left|T_{0}\right|=0$ (Ref. 9, Remark 8, p. 396).

A remark is needed here. Property $\left(\mathrm{P}_{2}\right)$ implies that the sets

$$
\tilde{Q}(t, y) \cap\left[E^{1} \times V(0, N)\right], \quad N \geq \sigma,
$$

are closed and convex, and hence the sets $\tilde{Q}(t, y)$ themselves are closed and convex. Conversely, if we assume that the sets $\tilde{Q}(t, y)$ are closed and convex, then the sets

$$
\tilde{Q}(t, y) \cap\left[E^{1} \times V(0, N)\right], \quad N \geq \sigma,
$$

are also closed and convex. Because of their special structure, property $(\mathrm{K})$ of the same sets

$$
\tilde{Q}(t, y) \cap\left[E^{1} \times V(0, N)\right]
$$

implies property (Q) of the same sets (Refs. 7, 10). Thus, if the sets $\tilde{Q}(t, y)$ are closed and convex, it suffices to require property $(K)$ in $\left(P_{2}\right)$ above. Finally, if we know that, for every $t_{0}$ fixed, $t_{0} \in G-T_{0},\left|T_{0}\right|=0$, we have $\left|f\left(t_{0}, y, u\right)\right| \rightarrow+\infty$ as $|u| \rightarrow \infty, u \in U\left(t_{0}, y\right)$, uniformly on every compact subset of $A\left(t_{0}\right)$, then the sets

$$
\tilde{Q}\left(t_{0}, y\right) \cap\left[E^{1} \times V(0, N)\right]
$$

certainly have property $(\mathrm{K})$ with respect to $y$ at every $\left(t_{0}, y_{0}\right), y_{0} \in A\left(t_{0}\right)[\mathrm{a}$ consequence of the continuity of $f_{0}\left(t_{0}, y, u\right), f(t, y, u)$ with respect to $\left.(y, u)\right]$. If the sets $\tilde{Q}\left(t_{0}, y\right)$ are closed and convex, then the sets

$$
\tilde{Q}\left(t_{0}, y\right) \cap\left[E^{1} \times V(0, N)\right]
$$

have also property (Q) with respect to $y$ at $\left(t_{0}, y_{0}\right)$ (Ref. 9, p. 395).

Property $\left(\mathbf{P}^{\prime}\right)$. The following variant of property $(\mathrm{P})$ is of interest (Ref. 10; see also Ref. 2). It will be expressed in terms of sequences of functions $y_{k}(t), t \in H, k=1,2, \ldots$ In applications, this will be any minimizing sequence of state functions.

We say that the sets $\tilde{Q}(t, y)$ satisfy property $\left(\mathrm{P}_{1}^{\prime}\right)$ provided, for any sequence $y_{k}(t), t \in G, k=1,2, \ldots$, with $y_{k}(t) \in A(t), y_{k}(t) \rightarrow y(t)$ strongly in 
$\left(L_{1}(G)\right)^{r}$, there are other functions $\mu(t), \mu_{k}(t), p(t), p_{k}(t), t \in G, k=$ $1,2, \ldots, \mu, \mu_{k} \in L_{1}(G), p, p_{k} \in\left(L_{1}(G)\right)^{r}$, such that

$$
\begin{aligned}
& \left(\mu_{k}(t), p_{k}(t)\right) \in \tilde{Q}\left(t, y_{k}(t)\right), \quad t \in G, \text { a.e., } k=1,2, \ldots, \\
& \mu_{k} \rightarrow \mu \quad \text { weakly in } L_{1}(G), \text { or } \mu_{k} \rightarrow \mu \text { in measure in } G, \\
& p_{k} \rightarrow p \quad \text { strongly in }\left(L_{1}(G)\right)^{r} \text { as } k \rightarrow \infty .
\end{aligned}
$$

We shall say that the sets $\tilde{O}(t, y)$ satisfy condition $\left(\mathrm{P}^{\prime}\right)$ provided the same sets satisfy conditions $\left(\mathrm{P}_{1}^{\prime}\right)$ and $\left(\mathrm{P}_{2}\right)$.

Control Spaces $U(t), V(\tau)$ Independent of the State Variables. When the control spaces $U(t)$ depend on $t$ only and $V(\tau)$ depend on $\tau$ only, the Nemitsky operators

$$
[y, u] \rightarrow f(\cdot, y(\cdot), u(\cdot)), \quad[\dot{y}, v] \rightarrow g(\cdot, \dot{y}(\cdot), v(\cdot)),
$$

and the analogous ones with $f_{0}, g_{0}$, have relevant properties which have been studied in detail in Refs. 11-13. Some of the results have been reported in Ref. 2.

On the basis of properties of the Nemitsky operators above, it has been proved in Refs. 12-13, for instance, that, if $y_{k} \rightarrow y$ in measure in $G$ and $u_{k}$ is bounded in the $L_{1}$-norm, say $\left\|u_{k}\right\| \leq L$ [or $f$ satisfies some natural analytic conditions as $(F)$, or $(G)$, or $(H)$ of Refs. 11-12], then the differences

$$
\delta_{k}(t)=f\left(t, y_{k}(t), u_{k}(t)\right)-f\left(t, y(t), u_{k}(t)\right)
$$

approach zero in measure in $G$ as $k \rightarrow \infty$. Then, the lower closure and lower semicontinuity theorems hold without requiring the verification that the sets $\tilde{Q}(t, y)$ have property $(\mathrm{Q})$, or $(\mathrm{P})$, or $\left(\mathrm{P}^{\prime}\right)$. We refer to Ref. 2 for properties $(\mathrm{F}),(\mathrm{G}),(\mathrm{H})$.

\section{Existence Theorem}

Let $p$ be given, $1 \leq p \leq \infty$, and let $q$ such that

$$
q^{-1}+p^{-1}=1 \text {. }
$$

A triple $(x, u, v)$ is said to be admissible provided $x \in S, u \in T, V \in \stackrel{\circ}{T}$, provided relations (4)-(5) hold, provided

$$
\begin{aligned}
f_{0}(t, \mathcal{M} x(t), u(t)) \in L_{1}(G), & g_{0}(\tau, \mathscr{K} x(\tau), v(\tau)) \in L_{1}(\Gamma), \\
f(t, \mathscr{M} x(t), u(t)) \in\left(L_{p}(G)\right)^{r}, & g(\tau, \mathscr{K} x(\tau), v(\tau)) \in\left(L_{p}(\Gamma)\right)^{r^{\prime}},
\end{aligned}
$$


and the state equation (3), or

holds for all $u \in W$.

$$
\mathscr{F} w=h(x, u, v) w,
$$

We shall consider below nonempty closed classes $\Omega$ of admissible systems $(x, u, v)$. A class $\Omega$ of admissible triples is said to be closed if the following occurs: if $\left(x_{k}, u_{u}, v_{k}\right) \in \Omega, k=1,2, \ldots, x_{k} \rightarrow x$ in $(X, \mathscr{T})$ as $k \rightarrow \infty$, $x \in S$, if $I\left[x_{k}, u_{k}, v_{k}\right] \rightarrow a<\infty$ as $k \rightarrow \infty$, and there are admissible triples $(x, u, v)$ such that $I[x, u, v] \leq a$, then there is also some triple $(x, \bar{u}, \bar{v}) \in \mathbf{\Omega}$ with $I[x, \bar{u}, \bar{v}] \leq a$. This definition is justified by lower closure theorems (Refs. 4, 6, 9, 14, 15).

For the operators $\mathscr{M}, \mathscr{K}, \mathscr{F}$ introduced above, we shall need the property of closure in $S$, the closure graph property in $S$, and the convergence property that we introduced in Section 7 of Ref. 2.

Given a nonempty class $\Omega$ of admissible triples $(x, u, v)$, we shall denote by $\Omega_{0}$ the nonempty subset of only those $(x, u, v) \in \Omega$ with $I[x, u, v] \leq M_{0}$ for some constant $M_{0}$. We shall also denote by $\Lambda_{0}, \Lambda, \AA$ the sets

$$
\begin{aligned}
\Lambda_{0} & =\{x\}_{\Omega_{0}}=\left\{x \in X \mid(x, u, v) \in \Omega_{0}\right\}, \\
\Lambda & =\{(x, u)\}_{\Omega_{0}}=\left\{(x, u) \in X \times T \mid(x, u, v) \in \Omega_{0}\right\}, \\
\AA & =\{(x, v)\}_{\Omega_{0}}=\left\{(x, v) \in X \times \stackrel{\circ}{T} \mid(x, u, v) \in \Omega_{0}\right\} .
\end{aligned}
$$

The vector functions $f(t, y, u), g(\tau, \dot{y}, v)$ actually define Nemitsky-type operators $F, G$ :

$$
\begin{aligned}
& F[x, u](t)=f(t, M x(t), u(t)), \quad t \in G, \\
& G[x, v](\tau)=g(\tau, K x(\tau), v(\tau)), \quad \tau \in \Gamma, \\
& F: \Lambda \rightarrow\left(L_{1}(G)\right)^{r}, \quad G: \AA \rightarrow\left(L_{1}(r)\right)^{r^{\prime}} .
\end{aligned}
$$

We shall need below the requirement that the images $F(\Lambda)$ of $\Lambda$ and $G(\AA)$ of $\AA$ are relatively sequentially weakly compact subsets of $\left(L_{1}(G)\right)^{r}$ and $\left(L_{1}(G)\right)^{r^{\prime}}$. This is certainly the case if $f_{0}, f$, and $g_{0}, g$ satisfy the following growth condition

(H) For $p=1$, we assume that, given any $\epsilon>0$, there are functions

$$
\phi_{\epsilon} \geq 0, \quad \phi_{\epsilon} \in L_{1}(G), \quad \text { and } \stackrel{\circ}{\epsilon}_{\epsilon} \geq 0, \quad \dot{\phi}_{\epsilon} \geq L_{1}(\Gamma),
$$

such that

$$
\begin{array}{ll}
|f(t, y, u)| \leq \phi_{\epsilon}(t)+\epsilon f_{0}(t, y, u) & \text { for all }(t, y, u) \in M . \\
|g(\tau, \dot{y}, v)| \leq \dot{\phi}_{\epsilon}(t)+\epsilon g_{0}(\tau, \dot{y}, v) & \text { for all }(\tau, \dot{y}, v) \in \stackrel{\circ}{M} .
\end{array}
$$

If $p>1$, we assume that there are functions

$$
\phi_{0} \geq 0, \quad \phi_{0} \in L_{1}(G), \quad \text { and } \stackrel{\circ}{\phi}_{0} \geq 0, \quad \dot{\phi}_{0} \in L_{1}(\Gamma),
$$


and constants $a>0, b>0$, such that

$$
\begin{aligned}
|f(t, y, u)|^{p} \leq \phi_{0}(t)+a f_{0}(t, y, u) & \text { for all }(t, y, u) \in M, \\
|g(\tau, \dot{y}, v)|^{p} \leq \dot{\phi}_{0}(t)+b g_{0}(\tau, \dot{y}, v) & \text { for all }(\tau, \dot{y}, v) \in \stackrel{\circ}{ } .
\end{aligned}
$$

This condition, for $p=1$, has been consistently used by Cesari (see, e.g., Refs. $6,9,14$ ), as a suitable extension of previous more restrictive growth hypotheses used by Tonelli and McShane.

We say that $f_{0}, f$ satisfy condition $(\alpha)$ on $G$ if there is some function $\psi(t) \geq 0, t \in G, \psi \in L_{1}(G)$ such that

$$
f_{0}(t, y, u) \geq-\psi(t)
$$

for all $(t, y, u) \in M$. We say that $f_{0}, f$ satisfy condition $(\beta)$ on $G$ if there are a function $\psi(t) \geq 0, t \in G, \psi \in L_{1}(G)$, and a constant $\gamma \geq 0$ such that

$$
f_{0}(t, y, u) \geq-\psi(t)-\gamma|f(t, y, u)|
$$

for all $(t, y, u) \in M$. Analogous properties hold for $g_{0}, g$ on $M$.

Finally, let $\mathscr{A}$ be an operator, not necessarily linear, from a set $S$ of a topological space $(X, \mathscr{T})$ into a topological space $Y=(Y, \mathscr{Z})$. We say that the operator

$$
\mathscr{A}: S \rightarrow(Y, \mathscr{Z}), \quad S \subset(X, \mathscr{T}),
$$

is closed on $S$, provided $x_{k} \in S, k=1,2, \ldots, x \in S, x_{k} \rightarrow x$ in $(X, \mathscr{T}), \mathscr{A} x_{k} \rightarrow y$ in $(Y, \mathscr{Z})$, implies $y=\mathscr{A} x$. We say that

$$
\mathscr{A}: S \rightarrow(Y, \mathscr{Z}), \quad S \subset(X, \mathscr{T}),
$$

has the closed graph property provided $x_{k} \in S, k=1,2, \ldots, x \in X, x_{k} \rightarrow x$ in $(X, \mathscr{T}), \mathscr{A} x_{k} \rightarrow y$ in $(Y, \mathscr{Z})$, implies $x \in S$ and $y=\mathscr{A} x$.

We say that the operator

$$
\mathscr{A}: S \rightarrow(Y, \mathscr{Z}), \quad S \subset(X, \mathscr{T}),
$$

has the convergence property [with respect to $S,(X, \mathscr{T}),(Y, \mathscr{Z})]$ provided, if $x_{k} \in S, k=1,2, \ldots, x \in X, x_{k} \rightarrow x$ in $(X, \mathscr{T})$, then the sequence $\mathscr{A} x_{k}, k=$ $1,2, \ldots$, has a convergent subsequence in $(Y, \mathscr{Z})$, that is, there is some $y \in T$ and a subsequence $\left[k_{s}\right]$ such that $x_{k_{s}} \rightarrow y$ as $s \rightarrow \infty$ in $(Y, \mathscr{Z})$.

If $S$ is the whole space $X$, then closure and closed graph properties are identical. In Ref. 2 we have given examples and criteria for some of these properties.

Theorem 3.1. Existence Theorem for Optimal Weak Solutions. Let us assume that $A, M, f_{0}, f$ satisfy conditions $(\mathrm{C})$ on $G$, that $B_{0}, \stackrel{\circ}{M}, g_{0}, g$ satisfy condition $(\mathrm{C})$ on $\Gamma$, that $f_{0}, f$ satisfy condition $(\beta)$ on $G$, and $g_{0}, g$ satisfy condition $(\beta)$ on $\Gamma$. Let us assume that the sets $\tilde{Q}(t, y)$ in $E^{r+1}$ satisfy 
property (Q) with respect to $y$ only at all $(t, y) \in A, t \in G-T_{0},\left|T_{0}\right|=0$, and the sets $\tilde{R}(\tau, \stackrel{o}{y})$ in $E^{r^{\prime}+1}$ satisfy property (Q) with respect to $\dot{y}$ only at all $(\tau, \dot{y}) \in B, \tau \in \Gamma-\dot{T}_{0}^{\circ}, \mu\left(\dot{T}_{0}\right)=0$. Let us assume that relation (1) holds, and that $\mathcal{M}, \mathscr{K}, \mathscr{F}$ have the closure property in $S$, that $\mathcal{M}$ and $\mathscr{K}$ have the strong convergence property in $S$, and that at least one of the operators $\mathscr{M}, \mathscr{K}, \mathscr{F}$ has the closure graph property. Let $\Omega$ be a nonempty closed class of admissible systems $(x, u, v)$ such that $\Lambda_{0}$ is sequentially relatively compact in $(X, \mathscr{T})$ and that the images $F(\Lambda)$ of $\Lambda$ and $G(\AA)$ of $\AA$ are relatively sequentially weakly compact subsets of $\left(L_{p}(G)\right)^{r}$ and $\left(L_{p}(\Gamma)\right)^{r^{\prime}}$, respectively. Then, the functional $I[x, u, v]$ has an absolute minimum in $\Omega$.

If $f_{0}, f$ satisfy condition $(\mathrm{H})$, then certainly $f_{0}, f$ satisfy condition $(\beta)$, and the image $F(\Lambda)$ of $\Lambda$ is a relatively sequentially weakly compact subset of $\left(L_{p}(G)\right)^{r}$. If $g_{0}, g$ satisfy condition $(H)$, then certainly $g_{0}, g$ satisfy condition $(\beta)$, and the image $G(\AA)$ of $\AA$ is a relatively sequentially weakly compact subset of $\left(L_{p}(\Gamma)\right)^{r^{\prime}}$.

Alternate Assumptions. Property (Q) above can be replaced by property $(\mathrm{P})$, or by property $\left(\mathrm{P}^{\prime}\right)$, for the sets $\tilde{Q}$, or $\tilde{R}$, or both. If the sets $U(t)$ depend on $t$ only, and one of the conditions $(\mathrm{F})$, or $(\mathrm{G})$, or $(\mathrm{H})$ holds for $f_{0}, f$ then conditions $(\mathrm{Q})$, or $(\mathrm{P})$, or $\left(\mathrm{P}^{\prime}\right)$ need not be verified. The same holds for the sets $V(\tau)$ and the functions $g_{0}, g$. Again, if $U(t)$ depends on $t$ only, and we know that, for $(x, u) \in \Lambda, u$ is bounded in norm, then the sets $\tilde{Q}(t, y)$, if convex and closed, need not verify conditions $(\mathrm{Q}),(\mathrm{P}),\left(\mathrm{P}^{\prime}\right)$, and $f_{0}$ need not verify conditions $(\mathrm{F}),(\mathrm{G}),(\mathrm{H})$. Analogous statements hold concerning $V(\tau)$, $\grave{\Lambda}, \tilde{R}(\tau, \stackrel{\circ}{y}), g_{0}$.

Remark 3.1. As often occurs, control variables may appear in the expressions of the operators $\mathscr{L}, \mathscr{F}, \mathcal{M}, \mathscr{K}$, and therefore in $\mathscr{F}$. Existence theorems analogous to Theorem 3.1 hold, as we have already shown in Ref. 3 , Section 5 . They are obtained by treating such controls as state variables. We shall see this situation in examples below (Section 4).

Conversely, it may occur that state variables, appearing in the functions $f_{0}, f, g_{0}, g$, are better treated as control variables (for instance, derivatives of state variables). Again, we shall see this situation in examples below.

Proof of Theorem 3.1. As usual, let $i$ denote the infimum of $I[x, u, v]$ in the class $\Omega$, and hence also in the class $\Omega_{0},-\infty \leq i \leq M_{0}<+\infty$. We write

$$
I[x, u, v]=I_{1}+I_{2}, \quad I_{1}=\int_{G} f_{0} d t, I_{2}=\int_{\Gamma} g_{0} d \mu .
$$

Let $\left(x_{k}, u_{k}, v_{k}\right), k=1,2, \ldots$, be a sequence of elements in $\Omega_{0}$ with

$$
I_{k}=I\left[x_{k}, u_{k}, v_{k}\right] \rightarrow i \quad \text { as } k \rightarrow \infty .
$$


Let $I_{1 k}, I_{2 k}$ be the values of $I_{1}, I_{2}$ computed on the elements $x_{k}, u_{k}, v_{k}$. Since

$$
x_{k} \in \Lambda_{0}=\{x\}_{\Omega_{0}} \subset S \subset X, \quad k=1,2, \ldots,
$$

and $\Lambda_{0}$ is relatively sequentially compact as a subset of $(X, \mathscr{T})$, there is a subsequence, say still $[k]$, and an element $x \in X$ such that $x_{k} \rightarrow x$ in $(X, \mathscr{T})$. Let $z_{k}, \check{z}_{k}$ denote the functions

$$
\begin{aligned}
& z_{k}(t)=f\left(t, y_{k}(t), u_{k}(t)\right) \in F(\Lambda), \quad t \in G, \\
& \check{z}_{k}(\tau)=g\left(\tau, \dot{y}_{k}(\tau), v_{k}(\tau)\right) \in G(\Lambda), \quad \tau \in \Gamma, \quad k=1,2, \ldots,
\end{aligned}
$$

where

$$
y_{k}=\mathscr{M} x_{k}, \quad \dot{\circ}_{k}=\mathscr{K} x_{k},
$$

and

$$
\begin{array}{ll}
y_{k}(t) \in A(t), & u_{k}(t) \in U\left(t, y_{k}(t)\right), \quad t \in G, \text { a.e., } \\
\dot{y}_{k}(\tau) \in B(\tau), & v_{k}(\tau) \in V\left(\tau, \dot{\circ}_{k}(\tau)\right), \quad \tau \in \Gamma, \mu \text {-a.e., } k=1,2, \ldots
\end{array}
$$

Since $F(\Lambda)$ and $G(\AA)$ are relatively sequentially weakly compact subsets of $\left(L_{p}(G)\right)^{r}$ and $\left(L_{p}(\Gamma)\right)^{r^{\prime}}$, there is a subsequence, say still $[k]$, and clements $z \in\left(L_{p}(G)\right)^{r}, \stackrel{o}{z} \in\left(L_{p}(\Gamma)\right)^{r^{\prime}}$, such that $z_{k} \rightarrow z$ weakly in $\left(L_{p}(G)\right)^{r}$ and $\stackrel{o}{z}_{k} \rightarrow \stackrel{\circ}{z}$ weakly in $\left(L_{p}(\Gamma)\right)^{r^{\prime}}$ as $k \rightarrow \infty$. Thus, $\left\|z_{k}\right\|_{1}$ and $\left\|\dot{z}_{k}\right\|_{1}$ are bounded. By property $(\beta)$, we have now

$$
\begin{array}{cc}
f_{0}\left(t, \mathcal{M} x_{k}(t), u_{k}(t)\right) \geq-\psi(t)-\gamma z_{k}(t), & t \in G, \\
g_{0}\left(\tau, \mathscr{K} x_{k}(\tau), v_{k}(\tau)\right) \geq-\dot{\psi}(\tau)-\gamma \dot{z}_{k}(\tau), & \tau \in \Gamma ;
\end{array}
$$

hence,

$$
I_{1 k} \geq-\int_{G} \psi d t-\gamma\left\|z_{k}\right\|_{1}, \quad I_{2 k} \geq-\int_{\Gamma} \stackrel{\circ}{\psi} d \mu-\gamma\left\|z_{k}\right\|_{1} .
$$

This proves that

$$
I\left[x_{k}, u_{k}, v_{k}\right]=I_{1 k}+I_{2 k}
$$

is bounded below, that is, $i$ is finite. If $w=\left(w_{1}, w_{2}\right)$ is any element of $W$, then by relations (1) we know that

$$
w_{1} \in\left(L_{q}(G)\right)^{r}, \quad w_{2} \in\left(L_{q}(\Gamma)\right)^{r^{\prime}} .
$$

Hence,

$$
\begin{aligned}
\int_{G} z_{k}(t) \cdot w_{1}(t) d t \rightarrow \int_{G} z(t) \cdot w_{1}(t) d t, \quad \int_{\Gamma} \check{z}_{k}(\tau) \cdot w_{1}(\tau) d \mu & \\
& \rightarrow \int \check{z}(\tau) \cdot w_{2}(\tau) d \mu
\end{aligned}
$$


as $k \rightarrow \infty$. Since

$$
\mathscr{F}\left(x_{k}\right) w=h\left(x_{k}, u_{k}, v_{k}\right) w
$$

for all $k$, we conclude that $\mathscr{F}\left(x_{k}\right) w$ converges as $k \rightarrow \infty$, namely,

$$
\left(\mathscr{F} x_{k}\right) w \rightarrow \int_{G} z \cdot w_{1} d t+\int_{\Gamma} \dot{z} \cdot w_{2} d \mu \quad \text { as } k \rightarrow \infty .
$$

We have assumed that $\mathcal{M}$ and $\mathscr{K}$ have the convergence property. Thus, $x_{k} \rightarrow x$ in $(X, \mathscr{T})$ implies that, for some subsequence, say still [ $\left.k\right]$, we have

$$
\mathscr{M} x_{k} \rightarrow y, \quad \mathscr{K} x_{k} \rightarrow \dot{y}
$$

for some

$$
y \in\left(L_{1}(G)\right)^{r}, \quad \grave{y} \in\left(L_{1}(\Gamma)\right)^{r} .
$$

Finally, we have assumed that the operators $M, \mathscr{K}, \mathscr{F}$ have the closure property in $S$, and at least one has the closed graph closure property in $S$. Thus, $x \in S$, and then

$$
M x=y, \quad \mathscr{K} x=\stackrel{\circ}{y},
$$

and

$$
(\mathscr{F} x) w=\int_{a} z \cdot w_{1} d t+\int_{\Gamma} \stackrel{z}{z} \cdot w_{2} d \mu \quad \text { for all } w=\left(w_{1}, w_{2}\right) \in W .
$$

Here, the sets $\tilde{Q}(t, y)$ have property $(Q)$ with respect to $y$ in $A(t)$ for almost all $t$, and the sets $\tilde{R}(\tau, \dot{y})$ have property $(\mathrm{Q})$ with respect to $\dot{y}$ in $B(\tau)$ for $\mu$-almost all $t$. Then, by lower closure theorem (7.1) of Ref. 14, there are elements $u \in T, v \in \stackrel{T}{T}$ such that

$$
\begin{gathered}
y(t) \in A(t), \quad u(t) \in U(t, y(t)), \quad z(t)=f(t, y(t), u(t)), \quad t \in G, \text { a.e., } \\
\dot{y}(\tau) \in B(\tau), \quad v(\tau) \in V(\tau, \dot{y}(t)), \quad \grave{z}(\tau)=g(\tau, \dot{y}(\tau), v(\tau)), \quad \tau \in \Gamma, \mu \text {-a.e., } \\
f_{0}(t, y(t), u(t)) \in L_{1}(G), \quad g_{0}(\tau, \dot{y}(\tau), v(\tau)) \in L_{1}(\Gamma), \quad I[x, u, v] \leq i,
\end{gathered}
$$

where

$$
y=\mathscr{M} x, \quad \stackrel{o}{y}=\mathscr{K} x ;
$$

and, by comparison with Eq. (6), we also have

$$
(\mathscr{F} x) w=h(x, u, v) w, \quad w \in W .
$$


Thus, $\operatorname{system}(x, u, v)$ is admissible; and, since $\Omega$ is closed, there is also some admissible system $(x, \bar{u}, \bar{v})$ in $\Omega$ with $I[x, \bar{u}, \bar{v}] \leq i$. The same system $(x, \bar{u}, \bar{v})$ belongs to $\Omega$, hence

$$
I[x, \bar{u}, \bar{v}] \geq i, \quad \text { and } \quad I[x, \bar{u}, \bar{v}]=i .
$$

Theorem 3.1 is thereby proved under the main hypotheses. The proof is the same under the alternate hypotheses, by using the corresponding lower closure theorems in Refs. 9 and 15.

\section{Examples}

Example 4.1. Let $G$ be an open bounded connected subset of $E^{\nu}$, $\nu \geq 1$, of class $K$. We are concerned with the minimum of a functional

$$
I[x, u]=\int_{G} f_{0}(t, x(t), \nabla x(t), u(t)) d t,
$$

$x, u$ scalars, with state equations which we wish to be a weak form of

$$
\sum_{i=1}^{\nu} \partial^{2} x /\left(\partial t^{i}\right)^{2}=f(t, x(t), \nabla x(t), u(t))
$$

and with constraints

$$
x(t) \in A(t), \quad u(t) \in U(t, x(t)) .
$$

Here, $x$ and $u$ are functions on $G$. Thus, $g_{0}=0$, we have no boundary conditions on $x$, we can take $g=0, \mathscr{J}=0, \mathscr{K}=0$, and need make no

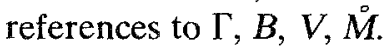

By introducing the increased control

$$
\tilde{u}(t)=\left(u^{1}, \ldots, u^{\nu}, u\right),
$$

we have the equivalent problem of minimizing the integral

$$
I[x, \tilde{u}]=\int_{G} f_{0}(t, x(t), \tilde{u}(t)) d t,
$$

with differential equations (8) in weak form and

$$
\partial x / \partial t^{i}=f_{i}=u^{i}, \quad i=1, \ldots, \nu,
$$

and constraints

$$
x(t) \in A(t), \quad \tilde{u}(t) \in E^{\nu} \times U(t, x(t)) .
$$

We shall think of $W$ as being $\left(C_{0}^{\infty}(G)\right)^{\nu+1}$, with

$$
w=(\tilde{w}, 0) \in W, \quad \tilde{w}=\left(w^{1}, \ldots, w^{\nu}, w\right), \quad \text { and } w^{1}, \ldots, w^{\nu}, w \in C_{0}^{\infty}(G) .
$$


As a weak form of the present system of differential equations, we now take

$$
\begin{aligned}
& \sum_{i=1}^{\nu} \int_{G}\left(\partial x / \partial t^{i}\right)\left(w^{i}(t)\right) d t-\int_{G} \sum_{i=1}^{\nu}\left(\partial x / \partial t^{i}\right)\left(\partial w / \partial t^{i}\right) d t \\
&=\int_{G} f(t, x(t), \tilde{u}(t)) w(t) d t+\sum_{i=1}^{\nu} \int_{G} u^{i}(t)\left(w^{i}(t)\right) d t
\end{aligned}
$$

for all

$$
\tilde{w}=\left(w^{1}, \ldots, w^{\nu}, w\right) \in\left(C_{0}^{\infty}(G)\right)^{\nu+1} .
$$

It is easy to verify that any strong solution $x, u$ of the original system of equations, say $x \in W_{1}^{2}(G), u \in T$, is certainly a solution of (12). Instead, we take $S=W_{1}^{1}(G)$ with the weak topology, thus $x \in S=W_{1}^{1}(G), u=T, p=1$, or equivalently $x \in S=W_{1}^{1}(G)$, and $\tilde{u}$ measurable in $G$.

We shall take in $W=\left(C_{0}^{\infty}(G)\right)^{\nu+1}$ the topology defined by the norm

$$
\|\tilde{w}\|_{W}=\max |w(t)|+\sum_{i=1}^{\nu} \max \left|\partial w / \partial t^{i}\right|+\sum_{i=1}^{\nu} \max \left|w^{i}(t)\right| .
$$

We have here $r=v+1$; and, if we denote by $\|\tilde{w}\|_{\infty}$ the norm of $\tilde{w}$ as an element of $\left(L_{\infty}(G)\right)^{\nu} \times W_{\infty}^{1}(G)$, then $\|\tilde{w}\|_{\infty}=\|\tilde{w}\|_{W}$ for every element $\tilde{w} \in W$. Also,

$$
\left(L_{\infty}(G)\right)^{r} \supset\left(L_{\infty}(G)\right)^{\nu} \times W_{\infty}^{1}(G) \supset W, \quad\left(L_{1}(G)\right)^{r} \subset W^{*},
$$

and relation (1) holds with $K=1$, since

$$
\|\tilde{w}\|_{\left(L_{\infty}(G)\right)^{r}} \leq\|\tilde{w}\|_{\infty}=\|\tilde{w}\|_{w} .
$$

We have here

$$
\mathcal{M} x=x, \quad \mathcal{M}: S \rightarrow L_{1}(G),
$$

and

$$
\mathscr{F}: S \rightarrow\left(L_{1}(G)\right)^{r} \subset W^{*}
$$

is the operator defined by the first member of (12). Now, if $x, x_{k} \in S=$ $W_{1}(G), k=1,2, \ldots$, and $x_{k} \rightarrow x$ weakly in $S=W_{1}^{1}(G)$, then $x_{k} \rightarrow x$ as $k \rightarrow \infty$ in $L_{1}(G)$, and

$$
\partial x_{k} / \partial t^{i} \rightarrow \partial x / \partial t^{i} \quad \text { as } k \rightarrow \infty \text { weakly in } L_{1}(G), \quad i=1, \ldots, \nu .
$$

Hence,

$$
\left(\mathscr{F} x_{k}\right) \tilde{w} \rightarrow(\mathscr{F} x) \tilde{w}
$$

for every

$$
\tilde{w} \in\left(L_{\infty}(G)\right)^{\nu} \times W_{\infty}^{1}(G)
$$


and then certainly for every

$$
\tilde{w} \in W=\left(C_{0}^{\infty}(G)\right)^{\nu+1} .
$$

Thus, $\mathscr{F}$ has the closure property in $S$ as well as the closed graph property in $S$. By Sobolev's imbedding theorems, $x_{k} \rightarrow x$ strongly in $L_{1}(G)$, or $M x_{k} \rightarrow M x$ strongly in $L_{1}(G)$. Thus, $\mathcal{M}: S \rightarrow L_{1}(G)$ has the strong convergence property as well as the closure and closure graph properties.

Note that here $R \subset E^{2 v+2}$ is the set of all $(t, y, \tilde{u})$ with

$$
t \in G, \quad y \in A(t), \quad \tilde{u} \in \tilde{U}(t, y)=E^{\nu} \times U(t, y),
$$

and that $f_{0}(t, y, \tilde{u}), \tilde{f}(t, y, \tilde{u})=\left(f_{1}, \ldots, f_{\nu}, f\right)$ are defined on $M$ with $f_{i}=u^{i}$, $i=1, \ldots, \nu, f=f(t, y, u)$. For $Z=\left(Z^{1}, \ldots, Z^{\nu}\right), \tilde{u}=\left(u^{1}, \ldots, u^{\nu}, u\right)$, the sets $\tilde{Q}(t, y)$ are the subsets of $E^{\nu+2}$ defined by

$$
\begin{aligned}
\tilde{Q}(t, y) & =\left[\left(z^{0}, z, Z\right) \mid z^{0} \geq f_{0}(t, y, \tilde{u}), z=f(t, y, \tilde{u}), Z^{i}=u^{i}, \tilde{u} \in \tilde{U}(t, y)\right] \\
& =\left[\left(z^{0}, z, Z\right) \mid z^{0} \geq f_{0}(t, y, Z, u), z=f(t, y, Z, u), u \in U(t, y), Z \in E^{\nu}\right] .
\end{aligned}
$$

Now, let $A(t)=E^{1}, U=E^{1}$,

$$
f_{0}=|t|^{\alpha}\left(x^{2}+|\nabla x|^{2}+u^{2}\right), \quad f=1+u+2^{-1}|u|, \quad 0<\alpha<\nu .
$$

Let us prove first that $f_{0}, f$ satisfy condition $(\mathrm{H})$ with $p=1$. Indeed, for every $0<\epsilon \leq 1$, we take

$$
\psi_{\epsilon}(t)=\epsilon^{-1}|t|^{-\alpha}+1 \geq 1, \quad \psi_{\epsilon} \in L_{1}(G)
$$

and we note that

$$
2^{-1}|u| \leq\left|u+2^{-1}\right| u|| \leq(3 / 2)|u|
$$

Now, either

$$
(3 / 2)|u| \geq \epsilon^{-1}|t|^{-\alpha}
$$

and then

$$
|f| \leq(3 / 2)|u|+1=(3 / 2)|u|^{-1} u^{2}+1 \leq(9 / 4) \in|t|^{\alpha} u^{2}+1 \leq \psi_{\epsilon}(t)+(9 / 4) \in f_{0}
$$

or

$$
(3 / 2)|u| \leq \epsilon^{-1}|t|^{-\alpha}
$$

and then again

$$
|f| \leq \psi_{\epsilon}(t)+(9 / 4) \epsilon f_{0} .
$$

An analogous statement holds for each function

$$
f_{i}=u^{i}, \quad i=1, \ldots, \nu .
$$


Let us prove that the class $\Lambda_{0}=\{x\}_{\Omega_{0}}$ is sequentially relatively compact in $W_{1}^{1}(G)$. It is enough to prove that, for $(x, u) \in \Omega_{0}$, the functions

$$
\Phi(t)=|x(t)|+\sum_{i=1}^{\nu}\left|\partial x / \partial t^{i}\right|, \quad t \in G,
$$

are equiabsolutely integrable in $G$. Indeed, either

$$
\Phi \geq \epsilon^{-1}|t|^{-\alpha},
$$

and then

$$
\Phi=\Phi^{-1} \Phi^{2} \leq \epsilon|t|^{\alpha}(\nu+1)\left(x^{2}+|\nabla x|^{2}\right) \leq \psi_{\epsilon}(t)+\epsilon(\nu+1) f_{0},
$$

or

$$
\Phi \leq \epsilon^{-1}|t|^{-\alpha},
$$

and then again

$$
\Phi \leq \psi_{\epsilon}(t)+\epsilon(\nu \in 1) f_{0} .
$$

Thus, $\Phi, f_{0}$ satisfy a growth condition $(\mathrm{H})$ with $p=1$, and again the functions $\Phi$ are equiabsolutely integrable in $G$.

For all $t \in G, t \neq 0$ (thus, certainly for almost all $t \in G$ ), the sets $\tilde{Q}(t, y)$ are closed and convex, and all functions $1, f, f_{i}=u^{i}, i=1, \ldots, \nu$, are of slow growth with respect to $f_{0}$ as $|u| \rightarrow \infty$. Thus, for almost all $t \in G$, the sets $\tilde{Q}(t, y)$ have property (Q) with respect to $y$ only in $A(t)=E^{1}$. However, property (Q) is not needed here. Indeed, for almost all $t \in G$, the $(\nu+1)$-vector function $\tilde{f}=\left(f_{i}, \ldots, f_{\nu}, f\right)$ has the property that $|\tilde{f}| \rightarrow+\infty$ as $|\tilde{u}| \rightarrow \infty$, uniformly for $y$ in any compact subset of $A(t)=E$. Thus, the convex closed sets $\tilde{Q}(t, y)$ have property $\left(\mathrm{P}_{2}\right)($ Section 2$)$. For the same sequence $\left[x_{k}\right]$ above, let us take

$$
\mu_{k}(t)=|t|^{\alpha} x_{k}^{2}(t), \quad p_{k}(t)=-1,
$$

so that

$$
\left(\mu_{k}(t), p_{k}(t)\right) \in Q\left(t, x_{k}(t)\right), \quad t \in G, \quad k=1,2, \ldots
$$

Here, $x_{k}(t) \rightarrow x(t)$ in $L_{1}$, and, therefore, by taking a suitable subsequence, also $x_{k}(t) \rightarrow x(t)$ in measure. Thus,

$$
|t|^{\alpha} x_{k}^{2}(t) \rightarrow|t|^{\alpha} x^{2}(t)
$$

in measure in $G$. Thus, property $\left(\mathrm{P}_{1}^{\prime}\right)$ holds, and $\left(\mathrm{P}^{\prime}\right)$ holds.

Example 4.2. This is the same as Example 4.1, with $A(t)=E^{1}$, $U=E^{1}, f_{0}(t, x, \nabla x, u), f(t, x, u)$ defined as follows. For $\tau \geq 0, \zeta \geq 0$, let 
$\Phi(\zeta, \tau)$ denote the real-valued continuous function defined by

$$
\begin{array}{cc}
\Phi(\zeta, \tau)=-\tau^{-1 / 2}+\zeta & \text { if } \tau \zeta^{2} \leq 4 \\
\Phi(\zeta, \tau)=2^{-2} \tau^{1 / 2} \zeta^{2} & \text { if } \tau \zeta^{2} \geq 4
\end{array}
$$

Thus,

$$
\Phi(\zeta, \tau) \geq \Phi(0, \tau)=-\tau^{-1 / 2} \quad \text { for all } \zeta \geq 0, \tau \geq 0
$$

Now, for

$$
x \in A(t)=E^{1}, \quad u \in U=E^{1}
$$

we take

$$
f(t, x, u)=u, \quad f_{0}(t, x, u)=\Phi(|\tilde{f}|,|t|),
$$

that is,

$$
f_{0}(t, x, \nabla x, u)=\Phi\left(\left(|\nabla x|^{2}+u^{2}\right)^{1 / 2},|t|\right) .
$$

Let us prove that $f_{0}, \tilde{f}$ satisfy property $(\mathrm{H})$ with $p=1$. For every $\zeta>0$, $\tau>0$ fixed, let us consider the function of $\epsilon$ only:

$$
\lambda(\epsilon)=-\epsilon^{-2} \tau^{-1 / 2}+\epsilon^{-1} \zeta, \quad 0<\epsilon \leq 1,
$$

which is continuous in $(0,1]$, with

$$
\lambda(0+)=-\infty, \quad \lambda(1)=-\tau^{-1 / 2}+\zeta,
$$

and thus $\lambda(\epsilon)$ has a maximum $\lambda_{\max }$ in $(0,1]$. For $\tau \zeta^{2} \leq 4, \lambda(\epsilon)$ is increasing in $(0,1]$; hence,

$$
\lambda_{\max }=\lambda(1)=-\tau^{-1 / 2}+\zeta
$$

for $\tau \zeta^{2} \geq 4$, we have

$$
\lambda_{\max }=\lambda\left(2 \tau^{-1 / 2} \zeta^{-1}\right)=2^{-2} \tau^{1 / 2} \zeta^{2} .
$$

Thus,

$$
-\epsilon^{-2} \tau^{-1 / 2}+\epsilon^{-1} \zeta \leq \Phi(\zeta, \tau)
$$

for all $0<\epsilon \leq 1, \zeta>0, \tau>0$, and the same relation holds actually for all $0<\epsilon \leq 1, \zeta \geq 0, \tau \geq 0$, and obvious conventions. With

$$
\psi_{\epsilon}(t)=\epsilon^{-1}|t|^{-1 / 2},
$$

we derive from (13) that

$$
\zeta=|\tilde{f}(t, x, u)| \leq \psi_{\epsilon}(t)+\epsilon f_{0}(t, x, \tilde{u}),
$$

and this relation holds for all $0<\epsilon \leq 1, t \in G, x \in A(t)$. Thus, condition $(\mathrm{H})$ is satisfied with $p=1$. 
Note that, for $\tau>0, \Phi(\zeta, \tau)$ is a continuous convex function of $\zeta$, $0 \leq \zeta<+\infty$, namely,

$$
\begin{aligned}
& \Phi(\zeta, \tau)=-\tau^{-1 / 2}+\zeta \quad \text { for } 0 \leq \zeta \leq 2 \tau^{-1 / 2} \\
& \Phi(\zeta, \tau)=2^{-2} \tau^{1 / 2} \zeta^{2} \quad \text { for } 2 \tau^{-1 / 2} \leq \zeta<+\infty
\end{aligned}
$$

For $t \neq 0$, the sets $\tilde{Q}(t, x)$ are closed and convex. Indeed, for fixed $t \neq 0$, the point $z=\left(z^{1}, \ldots, z^{\nu}, z^{\nu+1}\right)$, with $z^{1}=\bar{u}^{1}, \ldots, z^{\nu}=\bar{u}^{\nu}, z^{\nu+1}=u,(\bar{u}, u) \in$ $\tilde{U}=E^{\nu+1}$, describes $E^{\nu+1}$, while

$$
z^{0}=f_{0}(t, x, \tilde{u})=\Phi(|\tilde{u}|,|t|) .
$$

Again, as in Example (4.1), the sets $\tilde{Q}(t, y)$ satisfy property (Q). However, we do not need this property. Here, $|\tilde{f}(t, y, \tilde{u})| \rightarrow+\infty$ as $|\tilde{u}| \rightarrow+\infty$ uniformly for $x$ on every compact subset of $A(t)$, and the sets $\tilde{Q}(t, y)$ certainly have property $\left(\mathrm{P}_{2}\right)$. Here, $Q(t, x)=E^{v+1}$; and, for $p(t)=0$, certainly $p(t) \in Q(t, x)$ all $t \in G, x \in A(t)$. Moreover,

$$
-\tau^{-1 / 2} \leq T_{0}(z ; t, x) \leq 2^{-2} \tau^{1 / 2}+1
$$

for $|z| \leq 1$ and all $t \in G, x \in A(t)$. Thus, property $\left(\mathrm{P}_{1}\right)$ also holds. In other words, the sets $\tilde{Q}(t, x)$ satisfy property $(\mathrm{P})$.

Example 4.3. Let $G$ be an open bounded connected subset of $E^{\nu}$, $\nu \geq 1$, of class $K$. We are concerned with the minimum of a functional

$$
I[x, u]=\int_{G} f_{0}(t, x(t), \nabla x(t), u(t)) d t,
$$

$x$ scalar, $u$ an $m$-vector, with state equations which we wish to be a weak form of

$$
\begin{gathered}
\sum_{i=1}^{\nu} \partial^{2} x /\left(\partial t^{i}\right)^{2}=f_{1}(t, x(t), u(t)), \\
\sum_{i=1}^{\nu} A_{i}(t, x(t))\left(\partial x / \partial t^{i}\right)=f_{2}(t, x(t), u(t)),
\end{gathered}
$$

and with constraints

$$
x(t) \in A(t), \quad u(t)=\left(u^{1}, \ldots, u^{m}\right) \in U(t, x(t)) \subset E^{m} .
$$

As in Example 4.1,g=0, $\mathscr{J}=0, \mathscr{K}=0$, and we need make no reference to $\Gamma$, $B, V, \stackrel{M}{M}$. Here, the functions $A_{i}(t, x)$ are assumed to be continuous in cl $G \times E^{1}$.

By introducing the increased control

$$
\tilde{u}(t)=\left(\bar{u}^{1}, \ldots, \bar{u}^{\nu}, u^{1}, \ldots, u^{m}\right),
$$


and differential equations

$$
\partial x / \partial t^{i}=\vec{u}^{i}, \quad i=1, \ldots, \nu,
$$

we have the equivalent problem of minimizing the integral

$$
I[x, \tilde{u}]=\int_{G} f_{0}(t, x(t), \tilde{u}(t)) d t,
$$

with differential equations (15), (16), (18) in suitable weak form, and constraints (17). We shall think of $W$ as being $\left(C_{0}^{\infty}(G)\right)^{\nu+2}$, with $w=(\tilde{w}, 0) \in W, \quad \tilde{w}=\left(\bar{w}^{1}, \ldots, \bar{w}^{\nu}, w^{1}, w^{2}\right), \quad$ and $\quad \bar{w}^{1}, \ldots, w^{2} \in C_{0}^{\infty}(G)$.

As a weak form of the present system of differential equations, we now take

$$
\begin{aligned}
& \sum_{i=1}^{\nu} \int_{G}\left(\partial x / \partial t^{i}\right)\left(\bar{w}^{i}(t)\right) d t-\int_{G} \sum_{i=1}^{\nu}\left(\partial x / \partial t^{i}\right)\left(\partial w^{1} / \partial t^{i}\right) d t \\
& \quad+\int_{G}\left[\sum_{i=1}^{\nu} A_{i}(t, x(t))\left(\partial x / \partial t^{i}\right)\right] w^{2}(t) d t=\int_{G} f_{1}(t, x(t), u(t)) w^{1}(t) d t \\
& \quad+\int_{G} f_{2}(t, x(t), u(t)) w^{2}(t) d t+\sum_{i=1}^{\nu} \int_{G} \bar{u}^{i}(t) \bar{w}^{i}(t) d t
\end{aligned}
$$

for all

$$
\tilde{w}=\left(\bar{w}^{1}, \ldots, \bar{w}^{\nu}, w^{1}, w^{2}\right) \in\left(C_{0}^{\infty}(G)\right)^{\nu+2} .
$$

We take here $S=W_{2}^{1}(G)$ with the weak topology, thus, $x \in S=W_{2}^{1}(G)$, $u \in T, p=2$, or equivalently $x \in S=W_{1}^{1}(G), \tilde{u}$ measurable in $G$. We shall take in $W=\left(C_{0}^{\infty}(G)\right)^{\nu+2}$ the topology defined by the norm

$$
\|\tilde{w}\|_{W}=\max \left|w^{1}(t)\right|+\sum_{i=1}^{\nu} \max \left|\partial w^{1} / \partial t^{i}\right|+\max \left|w^{2}(t)\right|+\sum_{i=1}^{\nu} \max \left|\bar{w}^{i}(t)\right| .
$$

We have here $r=\nu+2$; and, if we denote by $\|\tilde{w}\|_{\infty}$ the norm of $\tilde{w}$ as an element of $W_{\infty}^{1}(G) \times\left(L_{\infty}(G)\right)^{\nu+1}$, then $\|\tilde{w}\|_{\infty}=\|\tilde{w}\|_{W}$ for every element $\tilde{w} \epsilon$ $W$. We have here $p=q=2$, and

$$
\|\tilde{w}\|_{\left.L_{2}(G)\right)^{r}} \leq\left\|w^{1}\right\|_{W_{2}^{1}(G)}+\left\|w^{2}\right\|_{L_{2}(G)}+\|\bar{w}\|_{\left(L_{2}(G)\right)^{\nu}} \leq|G|^{1 / 2}\|w\|_{W} .
$$

Hence,

$$
W \subset W_{2}^{1}(G) \times\left(L_{2}(G)\right)^{\nu+1} \subset\left(L_{2}(G)\right)^{r}
$$

and relation (1) holds with $K=|G|^{1 / 2}$.

Here,

$$
M x=x, \quad M: S \rightarrow L_{2}(G), \quad s=1, \quad r=2 .
$$


If $x_{k} \rightarrow x$ weakly in $S$, then $x_{k} \rightarrow x$ strongly in $L_{2}(G)$, or $M x_{k} \rightarrow M x$ strongly in $L_{2}(G)$, and thus $\mathcal{M}$ has the strong convergence property, and also the closure and closed graph properties in $S$.

Again, if $x_{k} \rightarrow x$ weakly in $S$, thus $\partial x_{k} / \partial t^{i} \rightarrow \partial x / \partial t^{i}$ weakly in $L_{2}(G)$, and $x_{k} \rightarrow x$ strongly in $L_{2}(G)$; then, for a suitable subsequence, say still $[k], x_{k} \rightarrow x$ in measure on $G, A_{i}\left(t_{1} x_{k}(t)\right) \rightarrow A_{i}(t, x(t))$ in measure in $G, i=1, \ldots, \nu$, because of the continuity of the functions $A_{i}$. If we know that

$$
z_{k}(t)=\sum_{i=1}^{\nu} A_{i}\left(t, x_{k}(t)\right)\left(\partial x_{k}^{i} / \partial t\right)
$$

converges weakly to some $z(t)$ in $L_{1}(G)$, then, by statement (9.2) of Ref. 2, we conclude that

$$
z(t)=\sum_{i=1}^{\nu} A_{i}(t, x(t))\left(\partial x^{i} / \partial t\right)
$$

We have proved that the operator $\mathscr{F}_{2}$ defined by the third integral in the first member of Eq. (19) has the closure and closed graph properties. Finally, the operator $\mathscr{F}_{1}$ defined by

$$
\left(\mathscr{F}_{1} x\right) w=\int_{G} \sum_{i=1}^{\nu}\left(\partial x / \partial t^{i}\right)\left(\partial w^{1} / \partial t^{i}\right) d t
$$

has the closure property as well as the closed graph property in $S=W_{2}^{1}(G)$ by the same argument as used for $\mathscr{F}$ in Example 4.1. Then, the operator $\mathscr{F}$ defined by the first member of Eq. (19) has the closed graph property, provided we know that $\left[z_{k}\right]$ converges weakly in $L_{1}(G)$.

We take here $A(t)=[-L, L]$ for some $L>0$ finite, $m=1, U(t, y)=E^{1}$, and

$$
f_{0}=|t|^{2}+|x|^{2}+|\nabla x|^{2}+|x||u|, \quad f_{1}=1+u, \quad f_{2}=x u .
$$

Here,

$$
\tilde{f}=\left(\bar{f}_{1}, \ldots, \bar{f}_{\nu}, f_{1}, f_{2}\right), \quad \bar{f}_{i}=\bar{u}^{i}, \quad i=1, \ldots, \nu, \quad f_{0} \geq 0,
$$

and thus $f_{0}, \tilde{f}$ satisfy conditions $(\alpha)$ and $(\beta)$ with $\psi=0, \gamma=0$.

Let $M_{1}>0$ be a given constant, and let $\Omega$ denote the set of all pairs $x, u, x \in W_{2}^{1}(G), u \in L_{2}(G)$, with

$$
\|u\|_{2} \leq M_{1}, \quad|x(t)| \leq L,
$$

satisfying Eqs. (17) and (19) and such that

$$
f_{0}(t, x(t), \nabla x(t), u(t)) \in L_{1}(G) .
$$

Let $M_{0}>0$ be a constant sufficiently large so that the subset $\Omega_{0}$ of all 
$(x, u) \in \Omega$ with $I[x, u] \leq M_{0}$ is nonempty. Then, for $(x, u) \in \Omega_{0}$, we certainly have $\|x\|_{2}^{1},\|\tilde{u}\|_{2},\|u\|_{2} \leq M_{2}$ for some constant $M_{2}$, and thus $\Lambda=\{x\}_{\Omega_{0}}$ is relatively weakly compact in $S=W_{2}^{1}(G)$, and

$$
\Lambda_{0}=\{\bar{u}(t), u(t), x(t) u(t)\}
$$

also is relatively weakly compact in $\left(L_{2}(G)\right)^{\nu+2}$, since $|x(t)| \leqslant L$. Then, some subsequence of $\left[z_{k}\right]$ is also weakly convergent in $L_{2}(G)$ as we required above.

Here, the subsets $\tilde{Q}(t, y)$ of $E^{\nu+3}$ are defined by

$\tilde{Q}(t, y)=\left[\left(z^{0}, \tilde{z}\right) \mid z^{0} \geq f_{0}(t, y, \tilde{u}), z^{1}=\bar{u}^{1}, \ldots, z^{\nu}=\bar{u}^{\nu}, z^{\nu+1}=u, z^{\nu+2}\right.$

where

$$
=x u, \tilde{u} \in \tilde{U}] \text {, }
$$

$\tilde{z}=\left(\bar{z}, z^{\nu+1}, z^{\nu+2}\right)=\left(z^{1}, \ldots, z^{\nu}, z^{\nu+1}, z^{\nu+2}\right), \tilde{u}=\left(\bar{u}, \ldots, \tilde{u}^{\nu}, u\right) \in \tilde{U}=E^{\nu+1}$,

and these sets are obviously closed and convex. They do not satisfy property (Q) at any $(t, y), t \in G, y=0$. Indeed, if we take

$$
\bar{u}=0, \quad t \in G, \quad x= \pm \eta, \quad \eta>0, \quad u= \pm \eta^{-1},
$$

we see that the points

$$
p^{ \pm}=\left[z^{0}=||^{2}+1, \bar{z}=0, z^{\nu+1}= \pm \eta^{-1}, z^{\nu+2}=1\right]
$$

belong to $\tilde{Q}(t, \pm \eta)$, respectively; hence, the point

$$
P=\left[z^{0}=|t|^{2}+1, \bar{z}=0, z^{\nu+1}=0, z^{\nu+2}=1\right]
$$

belongs to $\bigcup_{|y| \leq \eta} \tilde{Q}(t, y)$ for any $\eta>0$; and the same point $P$ belongs to $\bigcap_{\eta>0} \bigcup_{|y| \leqslant \eta} \tilde{Q}(t, y)$, while

$$
P \notin \tilde{Q}(t, y)=\left[z^{0} \geq|t|^{2}, \bar{z}=\bar{u}, z^{\nu+1}=u, z^{\nu+2}=0, \bar{u} \in E^{\nu}, \bar{u} \in E^{1}\right] .
$$

However, the sets $\tilde{Q}(t, y)$ satisfy property $\left(\mathrm{P}^{\prime}\right)$. To prove this, first we note that, if

$$
\left(x_{k}(t), \tilde{u}_{k}(t)\right), \quad t \in G, k=1,2, \ldots,
$$

is any minimizing sequence, we can always assume, by extraction, that $x_{k} \rightarrow x$ as $k \rightarrow \infty$ weakly in $S=W_{2}^{1}(G)$ to some $x \in W_{2}^{1}(G)$, and then $x_{k} \rightarrow x$ strongly in $L_{2}(G)$ by Sobolev's imbedding theorem. Now, we take

$$
\begin{aligned}
\bar{u}_{k}(t)=0, \quad u_{k}(t)=0, \quad \mu_{k}(t)=t^{2}+x_{k}^{2}(t), \quad \mu(t)=t^{2}+x^{2}(t), \\
p_{k}(t)=\left(\bar{u}_{k}(t), u_{k}(t), x_{k}(t) u_{k}(t)\right)=(0,0,0), \quad p(t)=(0,0,0), \quad t \in G, \\
k=1,2, \ldots,
\end{aligned}
$$

so that $\mu_{k} \rightarrow \mu$ strongly in $L_{1}(G), p_{k} \rightarrow p$ strongly in $\left(L_{1}(G)\right)^{\nu+2}$, and

$$
\left(\mu_{k}(t), p_{k}(t)\right) \in \tilde{Q}\left(t, x_{k}(t)\right), \quad t \in G, \quad k=1,2, \ldots
$$


Thus, property $\left(\mathrm{P}_{1}^{\prime}\right)$ is satisfied. Also we note that, for any $t \in G$, we have

$$
|\tilde{f}|=\left|\left(\bar{u}^{1}, \ldots, \bar{u}^{\nu}, u, x u\right)\right| \rightarrow+\infty
$$

as $|\tilde{u}| \rightarrow \infty$ uniformly for $x$ in any compact subset of $A(t)$. Thus, the sets $\tilde{Q}(t, x)$ satisfy property $\left(\mathrm{P}_{2}\right)$, and hence $\left(\mathrm{P}^{\prime}\right)$. We conclude that the specific problem under consideration has an absolute minimum.

Example 4.4. Let $G$ be a subset of the $t \xi$-space $E^{\nu+1}, \xi=\left(\xi^{1}, \ldots, \xi^{\nu}\right)$ of the form $G=(0, T) \times G^{\prime}$ is an open bounded connected subset of $E^{\nu}$ of class $K$. Thus, $\nu+1$ replaces $\nu$, and $\Gamma=\partial G^{\prime}$ is made up of parts

$$
\Gamma_{1}=\{0\} \times \mathrm{cl} G^{\prime}, \quad \Gamma_{2}=[0, T] \times \partial G^{\prime}, \quad \Gamma_{3}=\{T\} \times \mathrm{cl} G^{\prime} .
$$

On $\Gamma_{1}$ and $\Gamma_{3}$, we have the Lebesgue $\nu$-dimensional measure, or $\mid$ |, and we shall use the symbol $d \tau$ in integration. In $\Gamma_{2}$, we have the product measure $\sigma=|| \times \mu$ of the one-dimensional measure on $[0, T]$ and of the hyperarea $\mu$ on the boundary $\partial G^{\prime}$ of $G^{\prime}$, and we shall use the symbol $d t d \mu$ in integration. Given a function $x$ in $G$, say belonging to some Sobolev space $W_{p}^{l}(G)$, we shall denote by $\gamma x$ the boundary values, or traces, of $x$ on $\Gamma=\partial G$; specifically, we shall denote by $\gamma_{i} x$ the boundary values of $x$ on $\Gamma_{i}, i=1,2,3$. We also denote by $T, \stackrel{T}{i}_{i}$ the families of all measurable functions on $G, \Gamma_{i}$, $i=1,2,3$, respectively.

We are concerned with the minimum of a functional

$$
\begin{aligned}
I\left[x, u, v_{1}, v_{2}, v_{3}\right]=\int_{G} f_{0}(t, \xi, x(t, \xi),(\nabla x)(t, \xi), u(t, \xi)) d t d \xi \\
\\
\quad+\int_{\Gamma_{3}} g_{0}\left(\xi,\left(\gamma_{3} x\right)(\xi), v_{3}(\xi)\right) d \xi,
\end{aligned}
$$

with state equations (on $G$ and $\Gamma_{2}$ ) which will be a suitable weak form of the system of equations

$$
\begin{aligned}
& \partial x / \partial t-\sum_{i=1}^{m} \partial^{2} x /\left(\partial \xi^{i}\right)^{2}=f_{k}(t, \xi, x(t, \xi),(\nabla x)(t, \xi), u(t, \xi)) \text { in } G, \\
& A_{0}(t, \xi, x(t, \xi))(\partial x / \partial t)+\sum_{i=1}^{\nu} A_{i}(t, \xi, x(t, \xi))\left(\partial x / \partial \xi^{i}\right) \\
&= f_{2}(t, \xi, x(t, \xi),(\nabla x)(t, \xi), u(t, \xi)) \quad \text { in } G, \\
& \partial x / \partial n+v_{2}(t, \xi) \gamma_{2} x(t, \xi)=0 \quad \text { on } \Gamma_{2},
\end{aligned}
$$

and constraints

$$
\begin{array}{lll}
x(t, \xi) \in A(t, \xi), & u(t, \xi) \in U(t, \xi, x(t, \xi)) & \text { a.e.in } G \\
\left(\gamma_{3} x\right)(\xi) \in B(\xi), & v_{3}(\xi) \in V\left(\xi,\left(\gamma_{3} x\right)(\xi)\right) & \text { a.e. in } \Gamma_{3} .
\end{array}
$$


Here,

$$
x, u: G \rightarrow E^{1}, \quad v_{2}: \Gamma_{2} \rightarrow E^{1}, v_{3}: \Gamma_{3} \rightarrow E^{1}
$$

denote real-valued functions, $x$ state variable, $u, v_{2}, v_{3}$ controls. In other words, we are interested in the determination of a function $x(t, \xi)$ in $G$ [in particular, of its initial values, say $v_{1}(\xi)=x(0, \xi)$ on $\Gamma_{1}$, and of controls $u(t, \xi)$ in $G, v_{2}(t, \xi)$ on $\Gamma_{2}, v_{3}(\xi)$ on $\Gamma_{3}$, such that the functional (21) has its minimum value, under constraints (25), (26), and a suitable weak form of state equations (22), (23), and (24).

Above, the functions $f_{0}, f_{1}, f_{2}, g_{0}$ will be assumed to satisfy Carathéodory's condition $(\mathrm{C})$, though, in the actual examples, they will be taken to be continuous in their variables. The functions $A_{0}, A_{1}, \ldots, A_{\nu}$ are assumed to be continuous in their arguments, and uniformly Lipschitzian with respect to $x$. (In Example 4.5, we shall assume the same functions $A_{i}$ only continuous.)

By introducing the increased control

$$
\tilde{u}(t, \xi)=\left(\bar{u}^{1}, \ldots, \bar{u}^{\nu+1}, u\right),
$$

we have the equivalent problem of the minimu $n$ of the integral

$$
\begin{aligned}
I\left[x, \tilde{u}, v_{1}, v_{2}, v_{3}\right]=\int_{G} f_{0}(t, \xi, x(t, \xi), \tilde{u}(t, \xi)) d t d \xi \\
+\int_{\Gamma_{3}} g_{0}\left(\xi,\left(\gamma_{3} x\right)(\xi), v_{3}(\xi)\right) d \xi,
\end{aligned}
$$

with differential equations

$$
\begin{gathered}
\partial x / \partial t-\sum_{i=1}^{\nu} \partial^{2} x /\left(\partial \xi^{i}\right)^{2}=f_{1}(t, \xi, x(t, \xi), \tilde{u}(t, \xi)) \quad \text { in } G, \\
A_{0}(t, \xi, x(t, \xi))(\partial x / \partial t)+\sum_{i=1}^{\nu} A_{i}(t, \xi, x(t, \xi))\left(\partial x / \partial \xi^{i}\right) \\
=f_{2}(t, \xi, x(t, \xi), \tilde{u}(t, \xi)) \quad \text { in } G, \\
\partial x / \partial n+v_{2}(t, \xi) \gamma_{2} x(t, \xi)=0 \quad \text { on } \Gamma_{2}, \\
\partial x / \partial \xi^{i}=\bar{f}_{i}=\bar{u}^{i}, \quad i=1, \ldots, \nu, \quad \partial x / \partial t=u^{\nu+1} \quad \text { in } G,
\end{gathered}
$$

with constraints (25), (26), and

$$
u^{i} \in E^{1}, \quad i=1, \ldots, \nu+1 .
$$

We take for $W$ the space of all pairs $w=(\tilde{\omega}, \gamma \tilde{\omega})$, with

$$
\begin{array}{r}
\tilde{\omega}=\left(\bar{\omega}^{1}, \ldots, \bar{\omega}^{\nu+1}, \omega^{1}, \omega^{2}\right), \quad \bar{\omega}^{i} \in C_{0}^{\infty}(G), \quad i=1, \ldots, \nu+1, \\
\omega^{1} \in C^{\infty}(\mathrm{cl} G), \quad \omega^{2} \in C_{0}^{\infty}(\mathrm{cl} G) .
\end{array}
$$


Then,

$$
\gamma \tilde{\omega}=(0, \ldots, 0, \gamma \omega) \text {. }
$$

As a weak form of (22)-(24), we take the equation

$$
\begin{aligned}
\int_{G} \sum_{i=1}^{\nu} & \left(\partial x / \partial \xi^{i}\right)\left(\partial \omega^{1} / \partial \xi^{i}\right) d t d \xi+\int_{G}(\partial x / \partial t) \omega^{1}(t, \xi) d t d \xi \\
& +\int_{\Gamma_{2}} v_{2}(t, \xi) \gamma_{2} x(t, \xi) \gamma_{2} \omega^{1}(t, \xi) d t d \mu \\
& +\sum_{i=1}^{\nu} \int_{G}\left(\partial x / \partial \xi^{i}\right) \bar{\omega}^{i}(t, \xi) d t d \xi+\int_{G}(\partial x / \partial t) \bar{\omega}^{\nu+1}(t, \xi) d t d \xi \\
& +\int_{G}\left[A_{0}(t, \xi, x(t, \xi))(\partial x / \partial t)\right. \\
& \left.+\sum_{i=1}^{\nu} A_{i}(t, \xi, x(t, \xi))\left(\partial x / \partial \xi^{i}\right)\right] \omega^{2}(t, \xi) d t d \xi \\
= & \int_{G} f_{1}(t, \xi, x(t, \xi), \tilde{u}(t, \xi)) \omega^{1}(t, \xi) d t d \xi \\
& +\int_{G} f_{2}(t, \xi, x(t, \xi), \tilde{u}(t, \xi)) \omega^{2}(t, \xi) d t d \xi \\
& +\sum_{i=1}^{\nu+1} \int_{G} u^{i}(t, \xi) \bar{\omega}^{i}(t, \xi) d t d \xi
\end{aligned}
$$

for all

$$
\tilde{\omega}=\left(\bar{\omega}^{1}, \ldots, \bar{\omega}^{\nu+1}, \omega^{1}, \omega^{2}\right) \in\left(C_{0}^{\infty}(G)\right)^{\nu+1} \times\left(C^{\infty}(\mathrm{cl} G)\right)^{2} .
$$

Here, $\mathscr{F}_{w}$, or $\mathscr{F}\left(x, v_{2}\right) w$, that is, the operator $\mathscr{F}$ is defined by the first member of (27). It is easy to verify that any strong solution $x, u, v_{2}$ or (22)-(24), with $x \in W_{p}^{2}, u \in T, v_{2} \in L_{q}\left(\Gamma_{2}\right)$, is certainly a solution of (27) for all $w \in W$.

Note that, for every $x, v_{2}, \tilde{u}$ for which (27) holds for all $\tilde{\omega}$, that is, for all $\omega^{1}, \bar{\omega}^{i}, i=1, \ldots, \nu+1, \omega^{2}$ arbitrary, then necessarily

$$
\partial x / \partial t=\bar{u}^{\nu+1}, \quad \partial x / \partial \xi^{i}=\bar{u}^{i}, \quad i=1, \ldots, \nu+1, \quad \text { a.e. in } G,
$$

and Eq. (23) is satisfied also a.e. in $G$. We denote by $\mathscr{F}_{1}, \overline{\mathscr{F}}_{i}, i=1, \ldots, \nu+1$, $\mathscr{F}_{2}$ the single operators in the first member of (27) as expressed in terms of $\omega^{1}, \bar{\omega}^{i}, i=1, \ldots, \nu+1, \omega^{2}$, respectively.

Now, $M$ is here the set of all $(t, \xi, y, \tilde{u}) \in E^{2 v+4}$ with

$$
(t, \xi) \in \mathrm{cl} G=\mathrm{cl} G^{\prime} \times[0, T], \quad y \in A(t, \xi), \quad \tilde{u} \in \tilde{U}(t, \xi, y) .
$$

Let $f_{0}(t, \xi, y, \tilde{u}), f(t, \xi, y, \tilde{u})$ be real-valued continuous functions on $M$. 
Then, $\tilde{Q}(t, \xi, y)$ are the subsets of $E^{\nu+3}$ defined by

$$
\begin{aligned}
\tilde{Q}(t, \xi, y)= & {\left[\left(z^{0}, z, Z\right) \mid z^{0} \geq f_{0}(t, \xi, y, \tilde{u}), z=f(t, \xi, y, \tilde{u}), Z^{i}=u^{i},\right.} \\
& \tilde{u} \in \tilde{U}(t, \xi, y)] \\
= & {\left[\left(z^{0}, z, Z\right) \mid z^{0} \geq f_{0}(t, \xi, y, Z, u), z=f(t, \xi, y, Z, u),\right.} \\
& \left.u \in U(t, \xi, y), Z \in E^{\nu+1}\right],
\end{aligned}
$$

where

$$
Z=\left(Z^{1}, \ldots, Z^{\nu+1}\right) \quad \text { and } \tilde{u}=\left(u^{1}, \ldots, u^{\nu+1}, u\right) .
$$

We shall take here $S=W_{p}^{1}(G)$ with the weak topology, thus, $x \in S=$ $W_{p}^{1}(G), p \geq 2, u \in T, v_{2} \in \stackrel{\circ}{T}_{2}$, with $\stackrel{\circ}{T}_{2}$ a weakly closed subset of $L_{q}\left(\Gamma_{2}\right)$, $1 / p+1 / q=1$, which is bounded in the norm of $L_{q}\left(\Gamma_{2}\right)$. We take in $W$ the topology defined by the norm

$$
\begin{aligned}
\|w\|_{W}= & \|\left(\tilde{\omega}, \gamma \tilde{\omega} \|=\max \left|\omega^{1}(t, \xi)\right|+\max \left|\partial \omega^{1} / \partial t\right|+\sum_{i=1}^{\nu} \max \left|\partial \omega^{1} / \partial \xi^{i}\right|\right. \\
& +\max \left|\omega^{2}(t, \xi)\right|+\sum_{i=1}^{\nu} \max \left|\bar{\omega}^{i}(t, \xi)\right|,
\end{aligned}
$$

where all max are taken in cl $G$. With $r=\nu+2$, we have

$$
\begin{aligned}
\|\tilde{\omega}\|_{\left(L_{q}(G)\right)^{r}}+\|\gamma \tilde{\omega}\|_{L_{q}(\Gamma)} & \leq \omega^{1}\left\|_{W_{q}^{1}(G)}+\right\| \gamma \omega^{1}\left\|_{L_{q}(\Gamma)}+\sum_{i=1}^{\nu}\right\| \bar{\omega}^{i}\left\|_{L_{q}(G)}+\right\| \omega^{2} \|_{L_{q}(G)} \\
& \leq\left(|G|^{1 / q}+|\Gamma|^{1 / q}\right)\|w\|_{W} .
\end{aligned}
$$

From this we deduce that

$$
W \subset W_{q}^{1}(G) \times L_{q}(\Gamma) \times\left(W_{q}(G)\right)^{\nu+2} \subset\left(L_{q}(G)\right)^{r+1} \times\left(L_{q}(\Gamma)\right),
$$

and that relation (1) holds with

$$
K=|G|^{1 / q}+|\Gamma|^{1 / q}
$$

We have here

$$
\begin{gathered}
x \in S=W_{p}^{1}(G), \quad \mathcal{M} x=x, \quad \mathcal{M}: S \rightarrow L_{p}(G), \\
\mathscr{K} x=\gamma_{3} x, \quad \mathscr{K}: S \rightarrow L_{p}\left(\Gamma_{3}\right), \quad s=s^{\prime}=1 .
\end{gathered}
$$

Also, $x_{k} \rightarrow x$ weakly in $S$ implies $M x_{k} \rightarrow M x$ strongly in $L_{p}(G), \gamma_{2} x_{k} \rightarrow \gamma_{2} x$ strongly in $L_{p}\left(\Gamma_{2}\right), \mathscr{K} x_{k} \rightarrow \mathscr{K} x$ strongly in $L_{p}\left(\Gamma_{3}\right), \partial x_{k} / \partial t \rightarrow \partial x / \partial t, \partial x_{k} / \partial \xi^{i} \rightarrow$ $\partial x / \partial \xi^{i}$ weakly in $L_{p}(G), i=1, \ldots, \nu$. Furthermore, if $v_{2}, v_{2 k}, k=1,2, \ldots$, are elements of $\stackrel{\circ}{T}_{2} \subset L_{q}\left(\Gamma_{2}\right)$ with $v_{2 h} \rightarrow v_{2}$ weakly in $L_{q}\left(\Gamma_{2}\right)$, then the products $v_{2 k}\left(\gamma_{2} x_{k}\right)$ converge weakly in $L_{1}\left(\Gamma_{2}\right)$ to $v_{2}\left(\gamma_{2} x\right)$. Finally, from the definition of $\mathscr{F}_{1}\left(x_{2}, v_{2}\right)$, we conclude that

$$
\mathscr{F}_{1}\left(x_{k}, v_{2 k}\right) \omega^{1} \rightarrow \mathscr{F}_{1}\left(x, v_{2}\right) \omega^{1} \quad \text { as } k \rightarrow \infty \text { for every } \omega^{1} .
$$


Again, if we assume that $x_{k} \rightarrow x$ weakly in $S$, then $\overline{\mathscr{F}}_{i}\left(x_{k}\right) \bar{\omega}^{i} \rightarrow \overline{\mathscr{F}}_{i}(x) \bar{\omega}^{i}$, $i=1, \ldots, \nu+1$. Finally, if we assume that, for some constants $C, D$, we have

$$
\left|A_{i}(t, \xi, x)\right| \leq C|x|, \quad\left|A_{i}(t, \xi, x)-A_{i}(t, \xi, y)\right| \leq D|x-y|,
$$

for all $(t, \xi) \in G, x, y \in E^{1}$, then $x_{k} \rightarrow x$ strongly in $L_{p}(G), p \geqslant 2 \geqslant q \geqslant 1$, implies that $x_{k} \rightarrow x$ strongly in $L_{q}(G)$, and

$$
A_{i}\left(t, \xi, x_{k}(t, \xi)\right) \rightarrow A_{i}(t, \xi, x(t, \xi))
$$

strongly in $L_{q}(G)$. If, in addition,

$$
\partial x_{k} / \partial t \rightarrow \partial x / \partial t, \quad \partial x_{k} / \partial \xi^{i} \rightarrow \partial x / \partial \xi^{1}, \quad i=1, \ldots, \nu,
$$

weakly in $L_{p}(G)$, then the products appearing in the expression of $\mathscr{F}_{2}$ converge weakly in $L_{1}(G)$ toward the corresponding products, and

$$
\mathscr{F}_{2}\left(x_{k}\right) \omega^{2} \rightarrow \mathscr{F}_{2}(x) \omega^{2} .
$$

Thus, combining the various parts, we have

for every $w \in W$.

$$
\mathscr{F}\left(x_{k}, v_{2 k}\right) w \rightarrow \mathscr{F}\left(x, v_{2}\right) w
$$

We may take $p=q=2$,

$$
\begin{aligned}
f_{0} & =t^{2}+|\xi|^{2}+x^{2}+|\nabla x|^{2}+u^{2}, \\
f_{1} & =-1+t+|\xi|+x+u+2^{-1}|u|, \\
f_{2} & =(-2+t+|\xi|)^{2} x+u, \\
g_{0} & =\left(1+|\xi|^{2}\right) x^{2}+(1+|x|), \\
u \in U & =E^{1}, \quad v_{3} \in V_{3}=E^{1}, \quad v_{2} \in V_{2}=E^{1} .
\end{aligned}
$$

We take for $\Omega$ the set of all pairs $x, u, x \in W_{2}^{1}(G), u, v_{3}$ measurable, $v_{2} \in L_{2}(G),\left\|v_{2}\right\|_{2} \leq 1$, satisfying the constraints and the functional relations already mentioned. Note that the $\bar{u}^{i}, i=1, \ldots, \nu+1$, are assumed to be only measurable, but since the equations

$$
\partial x / \partial t=\bar{u}^{\nu+1}, \quad \partial x / \partial \xi^{i}=\bar{u}^{i}, \quad i=1, \ldots, \nu,
$$

hold a.e. in $G$, we see that

$$
\bar{u}^{i} \in L_{2}(G), \quad i=1, \ldots, \nu+1 .
$$

For $A_{0}, A_{1}, \ldots$, we may take functions as

$$
\begin{aligned}
& A_{0}=1, \quad A_{1},=|x|, \quad A_{2}=|t||x|, \\
& A_{3}=|x| \sin |x|, \quad A_{4}=|x|^{2}(1+|x|)^{-1},
\end{aligned}
$$


and analogous ones satisfying (28). Here, condition $(\mathrm{H})$ for $p=q=2$ is satisfied since

$$
\left|f_{1}\right|^{2},\left|f_{2}\right|^{2} \leq C+D f_{0}
$$

for suitable constants $C, D$.

The sets $\tilde{Q}(t, \xi, y)$ are obviously closed and convex. These sets satisfy property $(\mathrm{Q})$ (because $1, f_{1}, f_{2}$ are of slow growth with respect to $f_{0}$ ). However, this is not needed here. Indeed, for every $(t, \xi) \in G,|f| \rightarrow+\infty$ as $|u| \rightarrow+\infty$ uniformly for $x$ in any compact subset of $A(t, \xi)$. Moreover, if $\left[x_{k}\right]$ is any minimizing sequence of state functions, $x_{k} \rightarrow x$ as $k \rightarrow \infty$ weakly in $S=W_{2}^{1}(G)$, then, by Sobolev's imbedding theorem, $x_{k}, x \in L_{\lambda}(G), x_{k} \rightarrow x$ strongly in $L_{\lambda}(G)$ for any

$$
\lambda<2+(\nu+1)^{-1}
$$

If we take

$$
\begin{array}{cc}
\mu_{k}(t, \xi)=t^{2}+|\xi|^{2}+x_{k}^{2}(t, \xi), & \mu(t, \xi)=t^{2}+|\xi|^{2}+x^{2}(t, \xi), \\
p_{k}(t)=-1+t+|\xi|+x_{k}(t, \xi), & p(t)=-1+t+|\xi|+x(t, \xi),
\end{array}
$$

we see that $\mu_{k} \rightarrow \mu$ strongly in $L_{1}(G), p_{k} \rightarrow p$ strongly in $L_{1}(G)$, and that

$$
\left(\mu_{k}(t, \xi), p_{k}(t, \xi)\right) \in \tilde{Q}\left(t, \xi, x_{k}(t)\right), \quad t \in G, \quad k=1,2, \ldots,
$$

as we can see by taking $\bar{u}=0, u=0$. Thus, the sets $\bar{Q}(t, \xi, x)$ satisfy property $\left(\mathrm{P}^{\prime}\right)$. On $\Gamma_{3}, g=0, g_{0} \geq 0$, hence the corresponding sets $\tilde{R}(t, \xi, y)$ are half straight lines, and certainly convex and closed. The continuity of $g_{0}$ guarantees that property $(\mathrm{Q})$ is satisfied. The condition $(\mathrm{H})$ is also trivially satisfied. On $\Gamma_{1}, \Gamma_{2}$ we have both $g=0, g_{0}=0$, and no further discussion is needed.

If $i$ denotes the infimum of $I\left[x, \tilde{u}, v_{1}, v_{2}, v_{3}\right]$ in $\Omega$, and $\Omega_{0}$ denotes the subset of $\Omega$ with $I \leq i+1$, then $\|u\|_{2},\|\bar{u}\|_{2},\|x\|_{2}^{1} \leq C$ for some constant $C$, and the set $\Lambda=\{x\}_{\Omega_{B}}$ is certainly relatively sequentially weakly compact. The integral (21) has an absolute minimum in $\Omega$.

Note that the same argument above would hold if $f_{1}, f_{2}$ are replaced by

$$
\begin{aligned}
& f_{1}=\alpha_{1}(t, \xi, x)+\beta_{1}(t, \xi)(\nabla x)+\gamma_{1}(t, \xi, x) u, \\
& f_{2}=\alpha_{2}(t, \xi, x)+\beta_{2}(t, \xi)(\nabla x)+\gamma_{2}(t, \xi, x) u,
\end{aligned}
$$

with $\alpha_{1}, \alpha_{2}$ continuous functions in $\mathrm{cl} G \times E^{1}, \beta_{s}(t, \xi)=\left(\beta_{s 1}, \ldots, \beta_{s \nu}\right)$, $s=1,2,\left[\beta_{s i}\right]$ a $2 \times \nu$ matrix with entries continuous in $\mathrm{cl} G$, of rank 2 everywhere in $\mathrm{cl} G$, and $\gamma_{1}, \gamma_{2}$ continuous functions in $\mathrm{cl} G \times E^{1}$ with $\gamma_{1}^{2}+\gamma_{2}^{2}>0$ in cl $G \times E^{1}$.

Example 4.5. This is analogous to Example 4.4 and concerns the same problem (21)-(26). However, we do not assume condition (28) nor 
condition $(\mathrm{H})$. Here, the functions $A_{i}(t, \xi, x)$ are assumed only to satisfy Carathéodory's condition (C). Thus, we can take for $A_{0}, A_{1}, \ldots$, functions as

$$
\begin{array}{ccc}
A_{0}=t^{-\nu / 2}, \quad A_{1}=t^{-\nu}, & A_{1}=|x|^{2}, \\
A_{2}=|t||x|^{3}, \quad A_{3}=\exp (|x|), & A_{4}=t^{-1} \exp (|x|),
\end{array}
$$

and analogous ones. We may take here

$$
\begin{aligned}
f_{0} & =t^{2}+|\xi|^{2}+x^{2}+|\nabla x|^{2}+|x||u|, \\
f_{1} & =-1+t+|\xi|+x+u, \\
f_{2} & =(-2+t+|\xi|)^{2} x+x u, \\
g_{0} & =\left(1+|\xi|^{2}\right) x^{2}+(1+|x|) \quad \text { on } \Gamma_{3}, \\
u \in U & =E^{1}, \quad v_{3} \in V_{3}=E^{1}, \quad v_{2} \in V_{2}=E^{1} .
\end{aligned}
$$

We take for $\Omega$ the set of all pairs $x, \tilde{u}, x \in W_{2}^{1}(G), v_{3}$ measurable, $u \in L_{2}(G)$, $v_{2} \in L_{2}(G),\|u\|_{2} \leq L_{0},\left\|v_{2}\right\| \leq L_{1}$, with $L_{0}, L_{1}$ given constants, satisfying the constraints and the functional relations as in Example 4.4. Again, the $\vec{u}^{i}$, $i=1, \ldots, \nu+1$, are assumed only measurable; but, since

$$
\partial x / \partial t=\bar{u}^{\nu+1}, \quad \partial x / \partial \xi^{i}=\bar{u}^{i}, \quad i=1, \ldots, \nu, \quad \text { a.e. in } G,
$$

we that

$$
\bar{u}^{i} \in L_{2}(G), \quad i=1, \ldots, \nu+1 .
$$

Since $f_{0} \geq 0$, certainly $f_{0}, f_{1}, f_{2}$ satisfy properties $(\alpha)$ and $(\beta)$ with $\psi=0$ amd $\gamma=0$.

Let $i$ denote the infimum of $I$ in $\Omega$, and let $\Omega_{0}$ be the subset of $\Omega$ with

$$
I\left[x, \tilde{u}, v_{1}, v_{2}, v_{3}\right] \leq i+1 .
$$

Then, $\|x\|_{2},\|\nabla x\|_{2},\|u\|,\left\|r_{2}\right\| \leq C$ for some constant $C$, as well as $\|\bar{u}\|_{2} \leq C$ for elements $(x, \tilde{u})$ in $\Omega_{0}$. Thus, $\Lambda_{0}=\{x\}_{\Omega_{0}}$ is relatively weakly compact in $S=W_{2}^{1}(G)$. Since

$$
\|x\|_{2} \leq C, \quad\|\nabla x\|_{2} \leq C, \quad\|u\|_{2} \leq C,
$$

by Sobolev's imbedding theorem, we see that, from any sequence $\left[\left(x_{k}, \tilde{u}_{k}\right)\right]$ in $\Omega_{0}$, there is a subsequence, say still [ $\left.k\right]$, such that $x_{k} \rightarrow x$ strongly in $L_{2}(G)$, $u_{k} \rightarrow u$ weakly in $L_{2}(G)$, and hence $x_{u} u_{k} \rightarrow x u$ weakly in $L_{1}(G)$. This shows that $f_{1}(\Lambda), f_{2}(\Lambda)$ are relatively sequentially weakly compact in $L_{1}(G)$. We shall apply Theorem 3.1 with $p=1, q=\infty$. Relation (1) holds for $q=\infty$ by the same argument as in Example 4.4.

It remains to prove that $\mathscr{F}_{2}$ has the closure property, but this is a consequence of our statement 9.2 of Ref. 2 (the convergence property that 
we verified in Example 4.4 is not needed in Theorem 3.1). For any $(t, \xi) \in G$ and $X \in E^{1}$, obviously the sets $\tilde{Q}(t, \xi, x)$ are convex and closed. They do not satisfy property (Q) as it can be seen by the same argument as in Example 4.3. However, they have property $\left(\mathrm{P}^{\prime}\right)$ as proved for Example 4.3.

We conclude that the functional (21) has an absolute minimum in $\Omega$ in the specific case under consideration.

Example 4.6. This is analogous to Examples 4.4 and 4.5, but now we replace $\mathrm{Eq}$. (23) with the equation

$$
x \operatorname{div} x=f_{2}(t, \xi, x(t, \xi), \nabla x(t, \xi), u(t, \xi)) .
$$

The same arguments hold as for Examples 4.4 and 4.5 with $f_{0}, f_{1}, f_{2}, f_{0}$ chosen as stated there. The operator $\mathscr{F}_{2}$ becomes

$$
\mathscr{F}_{2}(x) w^{2}=\int_{G} x(t, \xi)\left[\partial x / \partial t+\sum_{i=1}^{\nu} \partial x / \partial \xi^{i}\right] w^{2}(t, \xi) d t d \xi,
$$

and the closure property of this operator was proved in Theorem 9.2 of Ref. 2.

\section{References}

1. CESARI, L., Geometric and Analytic Views in Existence Theorems for Optimal Control. I. Distributed Parameters, Journal of Optimization Theory and Applications, Vol. 14, pp. 505-520, 1974.

2. CESARI, L., Geometric and Analytic Views in Existence Theorems for Optimal Control.II. Distributed and Boundary Controls, Journal of Optimization Theory and Applications, Vol. 15, pp. 467-497, 1975.

3. CESARI, L., and COWLES, D. E., Existence Theorems in Multidimensional Problems of Optimization with Distributed and Boundary Controls, Archive for Rational Mechanics and Analysis, Vol. 46, pp. 321-355, 1972.

4. CESARI, L., Existence Theorems for Weak and Usual Optimal Solutions in Lagrange Problems with Unilateral Constraints, I and II. Transactions of the American Mathematical Society, Vol. 124, pp. 369-412 and 413-430, 1966.

5. CESARI, L., Existence Theorems for Optimal Controls of the Mayer Type, SIAM Journal on Control, Vol. 6, pp. 517-552, 1968.

6. CesARI, L., Closure, Lower Closure, and Semicontinuity Theorems in Optimal Control, SIAM Journal on Control, Vol. 9, pp. 287-315, 1971; and Erratum, SIAM Journal on Control, Vol. 11, p. 677, 1973.

7. KAISER, P. J., Seminormality Properties of Convex Sets, Rendiconti del Circolo Matematico di Palermo (to appear).

8. RuPP, R. D., Hypotheses Implying Cesari's Property (Q), Journal of Optimization Theory and Applications, Vol. 19, pp. 119-124, 1976. 
9. CeSARI, L., Lower Semicontinuity and Lower Closure Theorems without Seminormality Conditions, Annali di Matematica Pura ed Applicata, Vol.98, pp. 381-397, 1974.

10. Suryanarayana, M. B., Remarks on Lower Semicontinuity and Lower Closure, Journal of Optimization Theory and Applications, Vol. 19, pp. 125$140,1976$.

11. Cesari, L., and Suryanarayana, M. B., Convexity and Property (Q) in Optimal Control Theory, SIAM Journal on Control, Vol. 12, pp. 705-720, 1974.

12. Cesari, L., and SuryanarayanA, M. B., Closure Theorems Without Seminormality Conditions, Journal of Optimization Theory and Applications, Vol. 15, pp. 441-465, 1975.

13. Cesari, L., and SuryanARAYANA, M. B., Nemitsky's Operators and Lower Closure Theorems, Journal of Optimization Theory and Applications, Vol. 19, pp. 165-183, 1976.

14. CESARI, L., Closure Theorems for Orientor Fields and Weak Convergence, Archive for Rational Mechanics and Analysis, Vol. 55, pp. 332-356, 1974.

15. KaISER, P. J., and SuryanarayanA, M. B., Orientor Field Equations in Banach Spaces, Journal of Optimization Theory and Applications, Vol. 19, pp. 141-164, 1976. 